ARTICLE

Received 17 Feb 2015 | Accepted 14 Apr 2015 | Published 18 May 2015

DOI: $10.1038 /$ ncomms 168 OPEN

\title{
Trans-synaptic zinc mobilization improves social interaction in two mouse models of autism through NMDAR activation
}

\author{
Eun-Jae Lee ${ }^{1,2}$, Hyejin Lee ${ }^{2,3}$, Tzyy-Nan Huang ${ }^{4}$, Changuk Chung 2,3, Wangyong Shin ${ }^{2,3}$, \\ Kyungdeok Kim ${ }^{2,3}$, Jae-Young Koh ${ }^{5,6,7}$, Yi-Ping $\mathrm{Hsueh}^{4}$ \& Eunjoon Kim²,3
}

Genetic aspects of autism spectrum disorders (ASDs) have recently been extensively explored, but environmental influences that affect ASDs have received considerably less attention. Zinc $(Z n)$ is a nutritional factor implicated in ASDs, but evidence for a strong association and linking mechanism is largely lacking. Here we report that trans-synaptic $\mathrm{Zn}$ mobilization rapidly rescues social interaction in two independent mouse models of ASD. In mice lacking Shank2, an excitatory postsynaptic scaffolding protein, postsynaptic Zn elevation induced by clioquinol (a Zn chelator and ionophore) improves social interaction. Postsynaptic $\mathrm{Zn}$ is mainly derived from presynaptic pools and activates NMDA receptors (NMDARs) through postsynaptic activation of the tyrosine kinase Src. Clioquinol also improves social interaction in mice haploinsufficient for the transcription factor Tbr1, which accompanies NMDAR activation in the amygdala. These results suggest that trans-synaptic $\mathrm{Zn}$ mobilization induced by clioquinol rescues social deficits in mouse models of ASD through postsynaptic Src and NMDAR activation.

\footnotetext{
${ }^{1}$ Graduate School of Medical Science and Engineering, Korea Advanced Institute of Science and Technology, Daejeon 305-701, Korea. ${ }^{2}$ Center for Synaptic Brain Dysfunctions, Institute for Basic Science (IBS), Daejeon 305-701, Korea. ${ }^{3}$ Department of Biological Sciences, Korea Advanced Institute of Science and Technology, Daejeon 305-701, Korea. ${ }^{4}$ Institute of Molecular Biology, Academia Sinica, Taipei 115, Taiwan. ${ }^{5}$ Neural Injury Research Lab, University of Ulsan College of Medicine, Seoul 138-736, Korea. ${ }^{6}$ Asan Institute for Life Science, University of Ulsan College of Medicine, Seoul 138-736, Korea. ${ }^{7}$ Department of Neurology, University of Ulsan College of Medicine, Seoul 138-736, Korea. Correspondence and requests for materials should be addressed to Y.-P.H. (email: yph@gate.sinica.edu.tw) or to E.K. (email: kime@kaist.ac.kr).
} 
A utism spectrum disorders (ASDs) represent a neurodevelopmental disorder characterized by impaired social interaction and communication, and restricted and repetitive behaviour, interest and activity. ASDs affect $\sim 1 \%$ of the population and are thought to be strongly influenced by genetic factors. A large number of ASD-associated genetic variations have recently been identified, indicating that ASDs represent a genetically heterogeneous family of disorders ${ }^{1-3}$. Some of the genetic variations lie along common pathways/ functions, including synaptic transmission, transcriptional regulation and chromatin remodelling ${ }^{1-3}$. In addition, studies using mouse models of ASD carrying these mutations have begun to suggest possible mechanisms that may underlie the pathogenesis of ASD, namely glutamatergic dysfunction and an imbalance between excitatory and inhibitory synapses ${ }^{4-14}$.

Environmental influences, such as nutrition, toxins and poisons, drugs, infection and stress, are thought to have a significant influence on psychiatric disorders. In ASDs, wellknown examples of environmental influences include pre- or perinatal exposure to viruses or teratogens such as valproic acid and thalidomide ${ }^{15,16}$. However, studies on additional environmental influences and underlying mechanisms are at an early stage. This contrasts with the rapidly growing evidence for the contribution of genetic factors to ASDs. Because environmental factors are highly likely to interact with the genetic variations of ASD to determine the type, severity and trajectory of ASD symptoms, a balance between genetic and environmental causes is required in studies of ASDs.

Zinc $(\mathrm{Zn})$, the second-most abundant trace element with a critical role in human nutrition and health, regulates a variety of cellular processes and protein functions. Zn deficiency has been implicated in diverse neurological and psychiatric disorders, including Alzheimer's disease, Parkinson's disease, ASDs, attention deficit/hyperactivity disorder, schizophrenia, epilepsy and mood disorders ${ }^{17}$. The association of $\mathrm{Zn}$ with ASDs has been suggested based on its deficiency in individuals with ASDs, including a recent large cohort of 1,967 children $^{16,18}$, as well as the phenotypes of $\mathrm{Zn}$-deficient experimental animals ${ }^{19}$. This association is further supported by the potential therapeutic value of $\mathrm{Zn}$ supplementation in ASD treatment ${ }^{17,20}$. However, strong evidence supporting the association between $\mathrm{Zn}$ deficiency and ASDs is largely unavailable, and the mechanisms underlying the association remain obscure.

In the synapse, the main pool of $\mathrm{Zn}$ ions is presynaptic vesicles where $\mathrm{Zn}$ is in the millimolar range, whereas postsynaptic sites contain much smaller amounts of $\mathrm{Zn}$ (picomolar range) ${ }^{21-24}$. Presynaptic free $\mathrm{Zn}$ is co-released with glutamate during neuronal activity and serves to suppress NMDA receptors (NMDARs) in the synaptic cleft. Some $\mathrm{Zn}$ ions enter the postsynaptic sites through calcium channels, NMDARs and calcium-permeable AMPA receptors (AMPARs), and regulate target proteins such as NMDARs and TrkB receptors through mechanisms including those involving Src family tyrosine kinases (SFKs) ${ }^{25-27}$. Another important effector of postsynaptic $\mathrm{Zn}$ is Shank (also known as ProSAP), a family of excitatory postsynaptic scaffolding proteins with three known members (Shank1/2/3; refs 28,29). Zn binds to Shank $2 / 3$ and enhances their postsynaptic stabilization, promoting excitatory synapse formation and maturation ${ }^{30}$.

Shank2/3, members of the Shank family of postsynaptic scaffolding proteins (also known as ProSAP1/2), have been implicated in ASDs through human genetic studies ${ }^{31-36}$ and mouse model/cultured neuron studies ${ }^{19,30,37-48}$. Mice carrying Shank2/3 mutations display diverse dysfunctions at glutamate synapses ${ }^{40-46,49}$. One notable change is the reduction in NMDAR function observed in Shank $2^{-1-}$ mice (exons $6+7$ deletion $)^{45}$. In these mice, normalization of NMDAR function with an
NMDAR agonist (D-cycloserine) is associated with the rescue of impaired social interaction, suggesting that NMDAR hypofunction might underlie the social deficit in these mice. Although validation of this hypothesis will require further analyses, D-cycloserine has also been shown to rescue the impaired social interaction in mice with a haploinsufficiency of the transcription factor Tbr1 (T-box brain 1; ref. 50), which positively regulates the expression of Grin $2 b$ (ref. 51), encoding the GluN2B subunit of NMDARs.

In the present study, we demonstrate that trans-synaptic $\mathrm{Zn}$ mobilization by clioquinol, a $\mathrm{Zn}$ chelator and ionophore (termed CQ hereafter), rescues the social interaction deficits in Shank2 ${ }^{-/}$and Tbr1 ${ }^{+/-}$mice. CQ mobilizes $\mathrm{Zn}$ from enriched presynaptic pools to postsynaptic sites, where it enhances NMDAR function through Src activation. These results indicate that postsynaptic $\mathrm{Zn}$ rescues social interaction deficits in distinct mouse models of ASDs, and suggest that reduced NMDAR function is associated with ASDs.

\section{Results}

CQ rapidly improves social interaction in Shank2-/- mice. Based on the close associations among Zn, NMDAR, Shank and ASD mentioned above, we reasoned that $\mathrm{Zn}$ delivered to postsynaptic compartments might rescue the reduced NMDAR function and ASD-like behaviours observed in Shank2 ${ }^{-/-}$mice. To test this idea, we first intraperitoneally (i.p.) injected Shank2 $2^{-/}$mice with CQ $\left(30 \mathrm{mg} \mathrm{kg}^{-1}\right)$, a lipophilic Zn chelator $\left(K_{\mathrm{d}} \approx 10^{-7}\right)$ and ionophore that readily crosses the blood-brain barrier and mobilizes $\mathrm{Zn}$ down a concentration gradient ${ }^{52}$. We chose systemic administration of CQ because dietary $\mathrm{Zn}$ is known to have poor bioavailability and side effects including gastric irritation ${ }^{17,53}$.

Two hours after CQ treatment, the mice were subjected to the three-chamber social interaction test, which compares the preference of a mouse for a stranger mouse versus a novel inanimate object. We found that Shank2 $2^{-/}$mice displayed reduced social interaction compared with wild-type (WT) mice, as determined by time spent exploring/sniffing the target and the social preference indices derived from exploration time (see figure legend for details) (Fig. 1a-c and Supplementary Fig. 1a,d-i), consistent with previous results from untreated Shank $2^{-/-}$and WT mice ${ }^{45}$. This impairment was improved by CQ treatment. In contrast, social interaction in WT mice was not affected by CQ. Notably, Shank2 $2^{-/-}$mice in the chamber with a stranger often spent time in other activities such as jumping, contrary to WT mice, as reflected in the relatively low correlation between exploration time and chamber time (Supplementary Fig. 2), and showed an apparent lack of CQ-dependent improvement in social interaction, as determined by the time spent in chamber and the preference index derived from chamber time (Supplementary Fig. 1b,c).

When social novelty recognition was determined in the same three-chamber test, by measuring the preference for a previously encountered stranger mouse versus a new stranger mouse, Shank2 $2^{-1}$ mice showed levels of social novelty recognition comparable to those in WT mice (Fig. 1d-f and Supplementary Fig. $1 j-m)$, as reported previously ${ }^{45}$. In addition, CQ had no effect on social novelty recognition in both WT and Shank2 ${ }^{-/-}$mice.

The CQ-dependent rescue of social interaction in Shank2 ${ }^{-/-}$ mice but no effect of CQ on WT mice is unlikely attributable to differences in the amount of free $\mathrm{Zn}$ available for CQ binding in these mice, because total levels of free $\mathrm{Zn}$ measured with the fluorescent dye TFL-Zn were not different (Supplementary Fig. 3a,b). In addition, the levels of $\mathrm{Zn}$ transporter 3 (ZnT3), a protein required for $Z n$ transport into presynaptic vesicles ${ }^{54}$, 
a
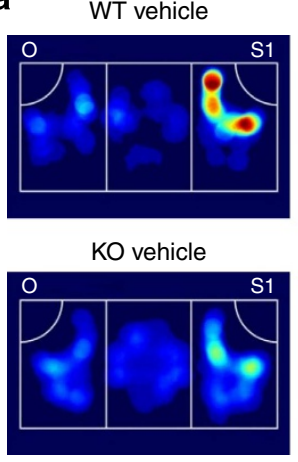

d
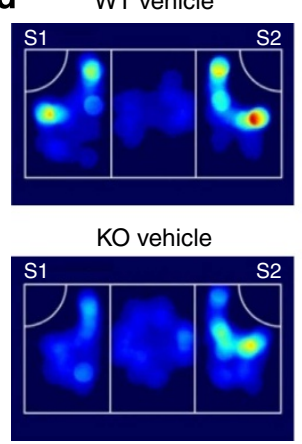

g

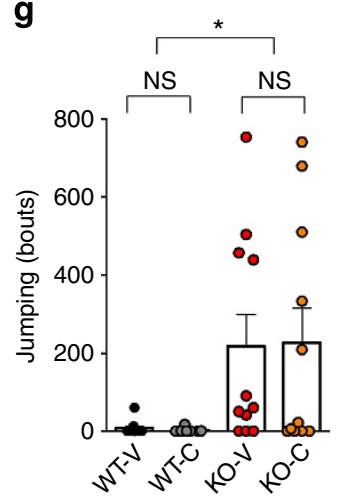

WT clioquinol

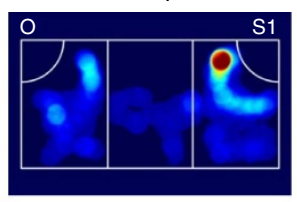

KO clioquinol

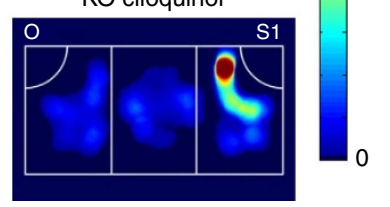

WT clioquinol

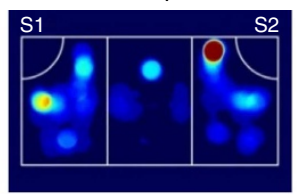

KO clioquinol

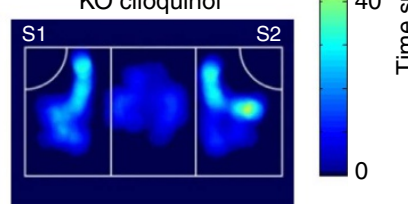

h

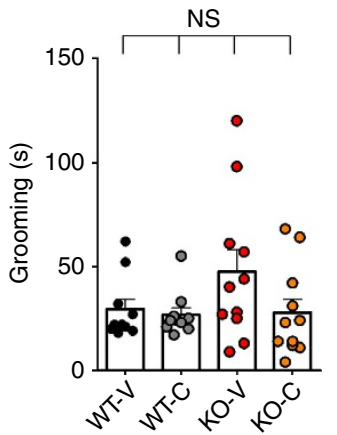

b
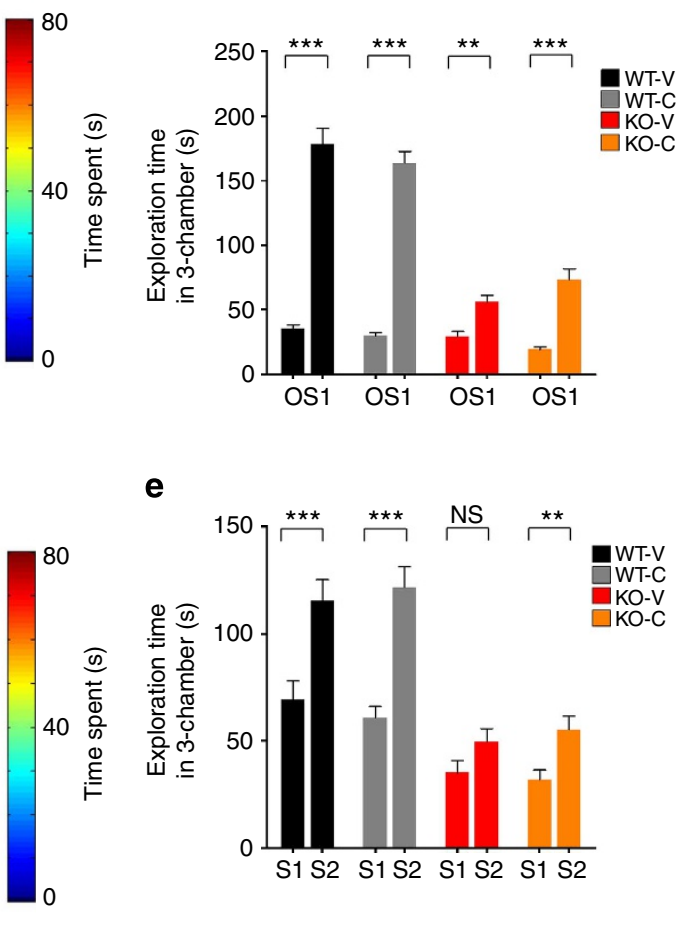

i
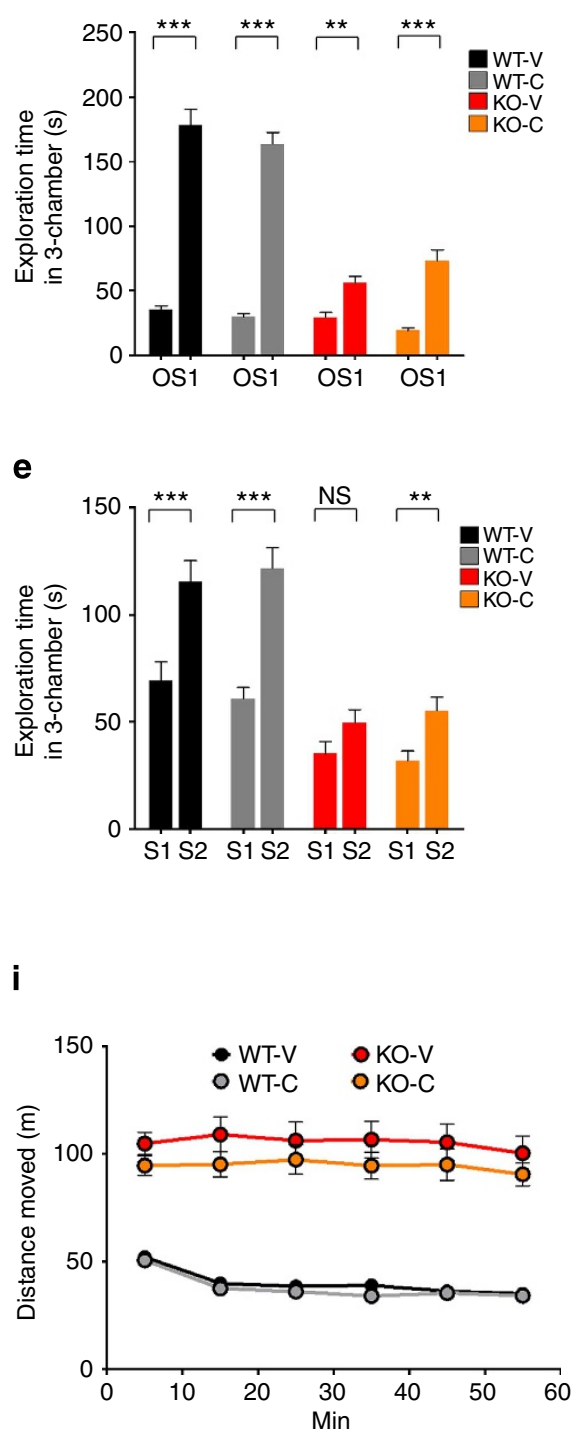

c

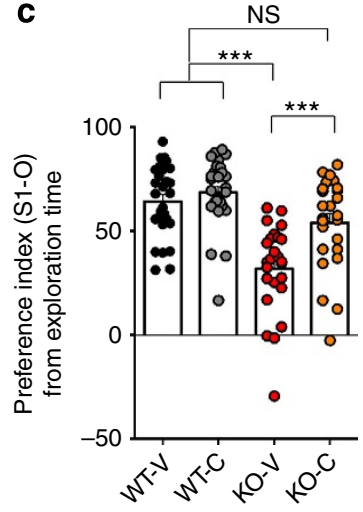

f

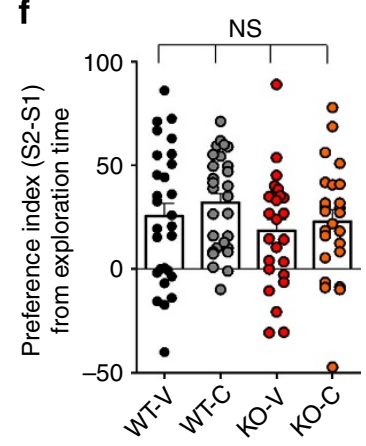

j

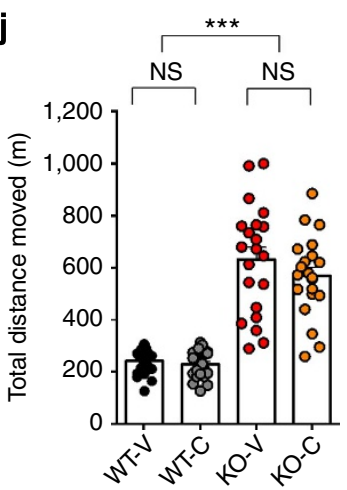

Figure 1 | CQ treatment rapidly improves social interaction in Shank2 ${ }^{-/-}$mice. (a-f) CQ improves social interaction in Shank2 ${ }^{-/-}$(KO) mice, but has no effect on WT mice (a-c). Note that levels of social novelty recognition are similar in WT and Shank $2^{-/-}$mice, and that CQ does not affect social novelty recognition in these mice (d-f). Mice were injected with CQ (30 $\mathrm{mg} \mathrm{kg}^{-1}$; i.p.), or vehicle, $2 \mathrm{~h}$ before behavioural tests. Heat maps in (a,d) represent examples of mouse movements. The social preference index from exploration time represents the numerical difference between the times spent exploring or sniffing the two targets (S1/stranger versus O/object or S2/new stranger versus $\mathrm{S} 1$ /previous stranger) divided by total time spent $\times 100$. $\mathrm{V}$, vehicle; C, CQ. ( $n=28$ for WT-V and WT-C, and 25 for $\mathrm{KO}-\mathrm{V}$ and $\mathrm{KO}-\mathrm{C}) ;{ }^{\star \star} P<0.01,{ }^{\star \star \star} P<0.001$; Kruskal-Wallis one-way analysis of variance (ANOVA) with Dunn's post hoc test). (g,h) CQ has no effect on jumping or grooming behaviour in Shank $2^{-/-}$mice. ( $n=10$ for WT-V and WT-C, and 11 for KO-V and KO-C, ${ }^{\star} P<0.05$, two-way ANOVA and Kruskal-Wallis one-way ANOVA with Dunn's post hoc test). (i,j) CQ does not normalize hyperactivity in Shank2 ${ }^{-/}$mice. ( $n=23$ for WT-V and WT-C, and 21 for KO-V and KO-C; ${ }^{\star \star \star} P<0.001$, two-way ANOVA and Kruskal-Wallis one-way ANOVA with Dunn's post hoc test). Data in all panels with error bars represent mean \pm s.e.m. NS, not significant.

which is the main pool of free $\mathrm{Zn}$ in the brain, were similar between genotypes (Supplementary Fig. 3c). Finally, whole-brain levels of $\mathrm{Zn}, \mathrm{Cu}$ or $\mathrm{Fe}$ were not different between WT and Shank2 $2^{-/-}$mice, which is in line with the above-mentioned TFL-Zn staining result, and, more importantly, 2-hr CQ treatment did not cause an acute reduction in the levels of these metals in WT or Shank2-/- mice (Supplementary Fig. 4a-c), suggesting that the chelating activity of CQ unlikely contributes to the observed social rescue.

In repetitive behaviour assays, vehicle-treated Shank2-/mice showed increased jumping behaviour but normal grooming in their home cages, relative to vehicle-treated WT mice, consistent with the previous results ${ }^{45}$; these behaviours were unaffected by CQ (Fig. 1g,h and Supplementary Fig. 5a-d). Similarly, CQ did not affect repetitive behaviours in WT mice.

In the open-field test, Shank2 ${ }^{-/-}$mice displayed increased locomotor activity relative to WT mice, as previously reported. This hyperactivity was not attenuated by CQ (Fig. 1i,j). Notably, Shank $2^{-/}$mice spent less time in the centre region of the open-field arena, a measure of anxiety-like behaviour. However, CQ had no effect on the centre-region time in these mice (Supplementary Fig. 5e). CQ did not affect the repetitive 
behaviour, locomotor activity or anxiety-like behaviour of WT mice. Together, these results suggest that CQ improves social interaction but has no effect on social novelty recognition, repetitive behaviour, hyperactivity or anxiety-like behaviour in Shank $2^{-/-}$mice.

NMDAR function at Shank2-/- synapses is restored by CQ. The CQ-dependent rescue of social interaction deficits in Shank $2^{-/-}$mice could involve normalization of the reported reduction in NMDAR function in these mice ${ }^{45}$. Consistent with this possibility, we found that CQ treatment restored normal levels of NMDAR function at Shank $2^{-/-}$hippocampal Schaffer collateral-CA1 pyramidal (SC-CA1) synapses, as determined by the ratio of NMDAR- to AMPAR-evoked excitatory postsynaptic currents (NMDA/AMPA ratio of eEPSCs; Fig. 2a). CQ, however, had no effect at WT synapses. In addition, CQ reversed the reduced tetanus-induced long-term potentiation (LTP), known to require NMDAR activity, at Shank2-/- SC-CA1 synapses, but had no effect on LTP at WT synapses (Fig. 2b). These results indicate that CQ restores NMDAR function at Shank2-/hippocampal SC-CA1 synapses.

We next measured the time course of CQ-dependent NMDAR activation at Shank $2^{-/-}$synapses. In these experiments, we treated Shank2-/- hippocampal slices with CQ for $20 \mathrm{~min}$ (in contrast to the continuous bath application for experiments described above) while monitoring NMDAR-mediated field excitatory postsynaptic potentials (NMDA-fEPSPs) before, a
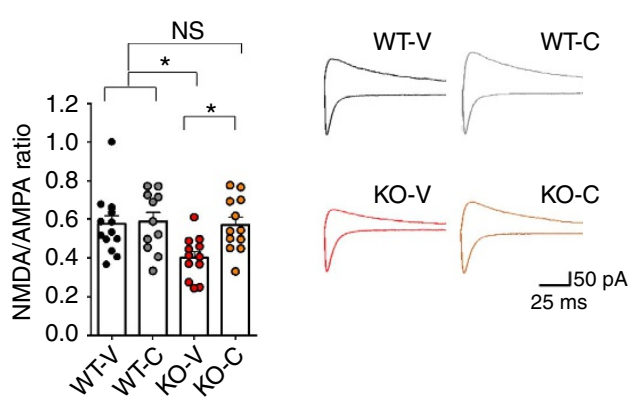

C

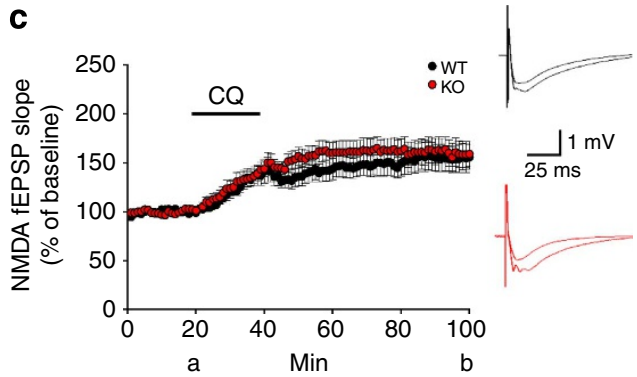

b
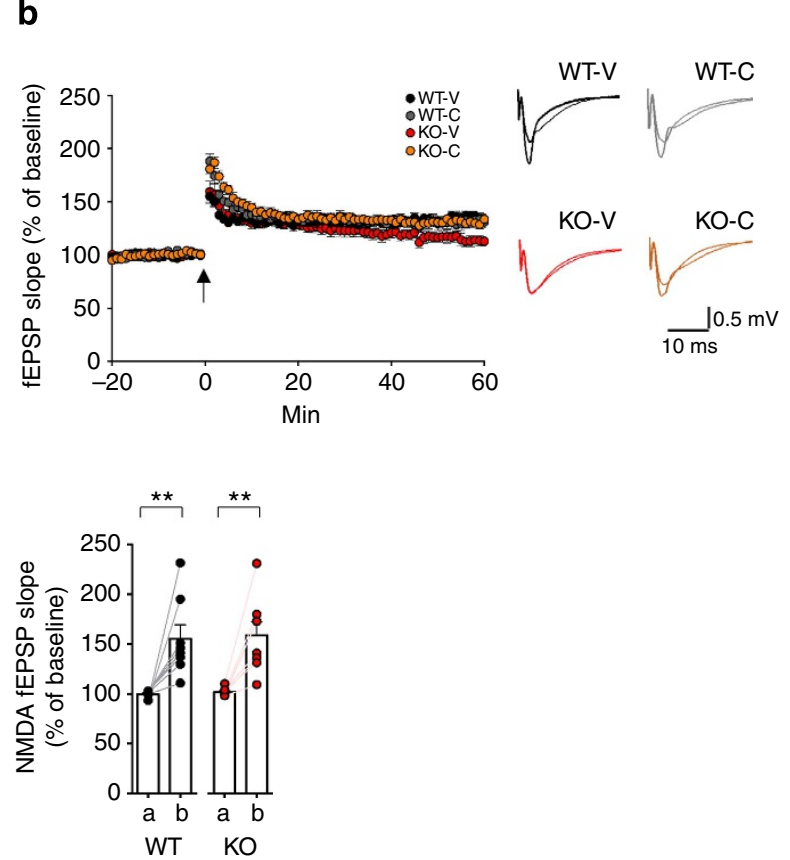

d

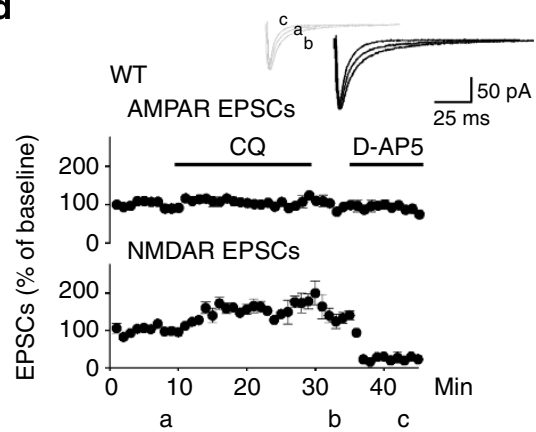

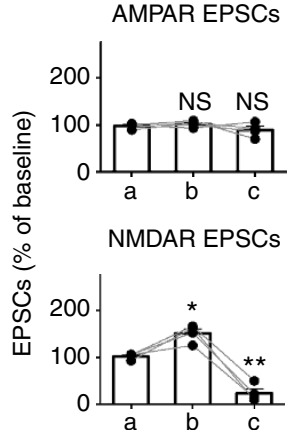

e

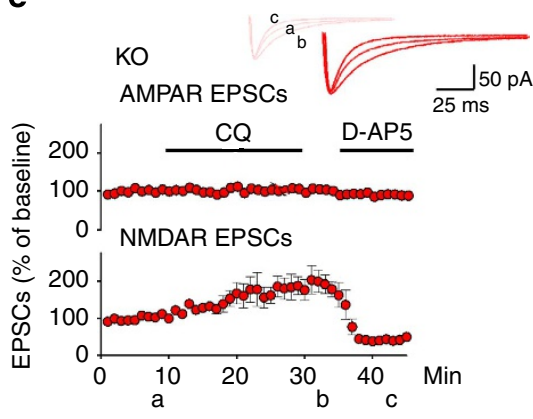

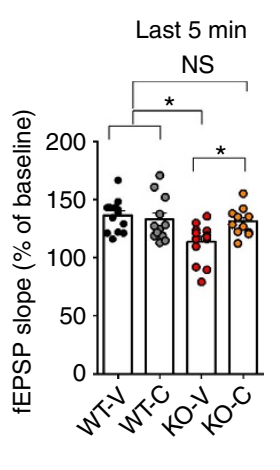

NS

$$
\frac{1}{4}
$$

Figure 2 | CQ restores NMDAR function at Shank2 ${ }^{-/-}$synapses. (a) CQ $(4 \mu M)$ normalizes the NMDA/AMPA ratio at Shank2 ${ }^{-/-}$hippocampal SC-CA1 synapses (P17-25), as measured by NMDA- and AMPA-eEPSCs. Representative eEPSC traces recorded at -70 and $+40 \mathrm{mV}$. NMDA-eEPSCs were measured at $+40 \mathrm{mV}$ holding potential, $60 \mathrm{~ms}$ after the stimulation. ( $n=14$ cells (from 10 animals) for WT-V, 11 (7) for WT-C, 12 (8) for KO-V and 12 (7) for KO-C, ${ }^{\star} P<0.05$; one-way analysis of variance (ANOVA) with Tukey's post hoc test). (b) CQ (4 $\left.\mu \mathrm{M}\right)$ restores LTP, induced by tetanus (100 Hz), at Shank2 ${ }^{-/-}$hippocampal SC-CA1 synapses (3-5 weeks), as measured by fEPSPs. ( $n=13$ slices (from 8 animals) for WT-V, 12 (5) for WT-C, 13 (7) for KO$\checkmark$ and 11 (5) for KO-C; ${ }^{\star} P<0.05$; one-way ANOVA with Tukey's post hoc test). (c) CQ ( $4 \mu \mathrm{M}, 20$ min) enhances NMDAR function at WT and Shank2 ${ }^{-/-}$ hippocampal SC-CA1 synapses, as measured by NMDA-fEPSPs. ( $n=8$ slices (from 7 animals) for WT and 8 (7) for KO; ${ }^{\star \star} P<0.01$; Student's $t$-test) The labels $a$ and $b$ indicate 5 -min duration before $C Q$ and the end of recording, respectively. (d,e) CQ ( $4 \mu \mathrm{M}, 20$ min) enhances NMDAR function at WT and Shank2-/- hippocampal SC-CA1 synapses, as determined by simultaneous measurements of NMDA- and AMPA-eEPSCs at - $40 \mathrm{mV}$. NMDA-eEPSCs were measured at $60 \mathrm{~ms}$ after stimulation. D-AP5 (50 $\mu \mathrm{M}, 10 \mathrm{~min})$ was used to test NMDAR dependence. The labels (a-c) indicate 5-min duration before and after $\mathrm{CQ}$, and at the end of recording, respectively. ( $n=4$ cells (three slices) for WT and 5 cells (four slices) for $\mathrm{KO}$; ${ }^{\star} P<0.05,{ }^{\star \star} P<0.01,{ }^{\star \star \star} P<0.001$; Student's t-test, compared with the 5-min duration before CQ). Data in all panels with error bars represent mean \pm s.e.m. NS, not significant. 
during and after CQ treatment. With CQ treatment, the initial slopes of NMDA-fEPSPs at Shank2-l- SC-CA1 synapses gradually increased to $\sim 150 \%$ of baseline levels and remained elevated, a response similar to that observed in WT slices (Fig. 2c). In contrast to NMDA-fEPSPs, AMPAR-mediated fEPSPs (AMPA-fEPSPs) were not affected by CQ treatment (Supplementary Fig. 6a). CQ also had no effect on AMPARrelated input-output ratio (AMPA-fEPSP slopes plotted against fibre volleys) or paired-pulse ratio at SC-CA1 synapses (Supplementary Fig. 6b,c).

To further confirm the CQ-dependent NMDAR activation, we simultaneously measured NMDA- and AMPA-eEPSCs using patch-clamp recordings. At a holding potential of $-40 \mathrm{mV}$, CQ increased NMDA-eEPSCs at Shank2 ${ }^{-1}$ SC-CA1 synapses, a result similar to that observed at WT synapses (Fig. 2d,e). The NMDAR antagonist D-AP5 significantly reduced NMDA-eEPSCs but not AMPA-eEPSCs, indicating that these events are NMDAR dependent. In contrast, AMPA-eEPSCs were not affected by CQ treatment (Fig. 2d,e). Consistent with this, the NMDA/AMPA ratios derived from these currents were increased in both genotypes (Supplementary Fig. 6d,e). Taken together, these results indicate that CQ enhances NMDAR but not AMPAR function at Shank $2^{-/-}$and WT synapses.

CQ mobilizes Zn from pre- to postsynaptic sites. Next, we determined whether CQ-dependent NMDAR activation requires $\mathrm{Zn}$. To test this, we used two different $\mathrm{Zn}$ chelators with much higher affinities for Zn than CQ: Ca-EDTA $\left(K_{\mathrm{d}} \approx 10^{-13}\right)$, which is membrane impermeable, and TPEN $\left(K_{\mathrm{d}} \approx 10^{-15}\right)$, which is membrane permeable. Preincubation of slices with Ca-EDTA before CQ treatment eliminated the CQ-dependent increase in NMDAR activity at Shank2 $2^{-1-}$ SC-CA1 synapses, as measured by NMDA-fEPSPs (Fig. 3a). In a control experiment, Ca-EDTA by itself had no effect on NMDA-fEPSPs (Supplementary Fig. 7a), as reported previously ${ }^{24,55}$. TPEN also blocked CQ-dependent NMDAR activation (Fig. 3b), although TPEN by itself caused a small increase in the basal activity of NMDARs (Supplementary Fig. 7b). Collectively, these findings suggest that CQ requires $\mathrm{Zn}$ for NMDAR activation. In addition, the absence of an effect of Ca-EDTA alone on NMDA-fEPSPs suggests that CQ-dependent NMDAR activation is unlikely the result of a

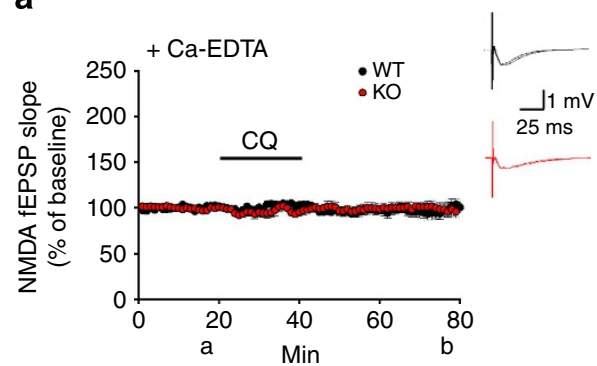

C

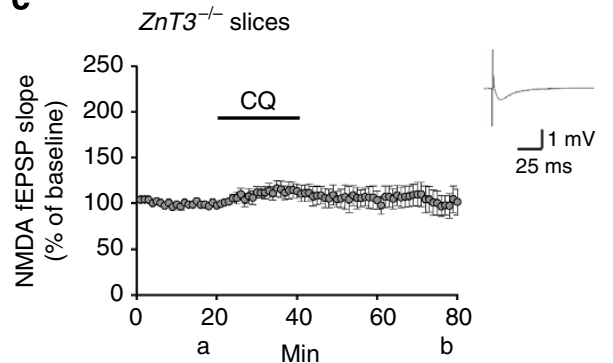

e

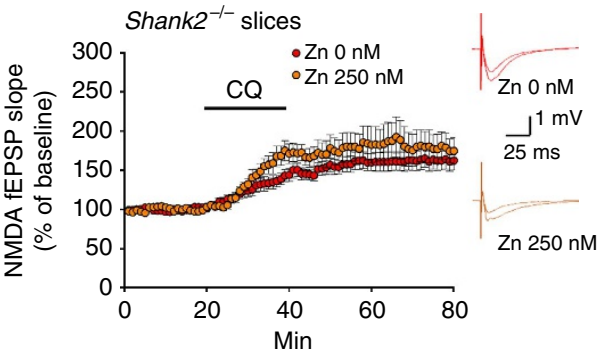

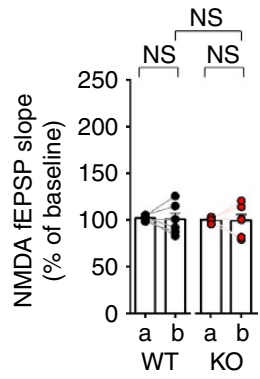

b
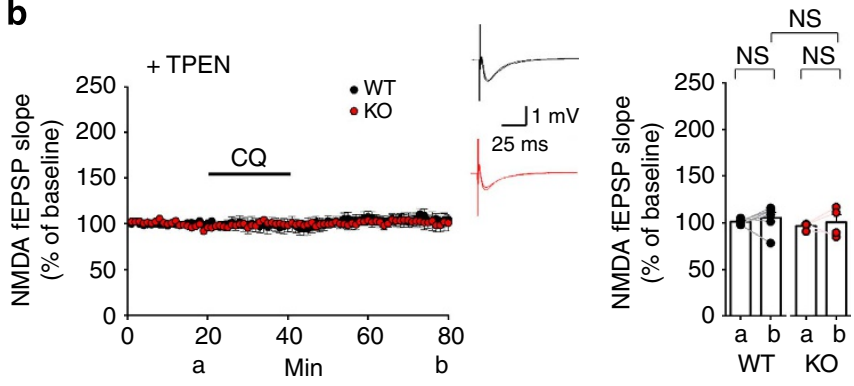

d
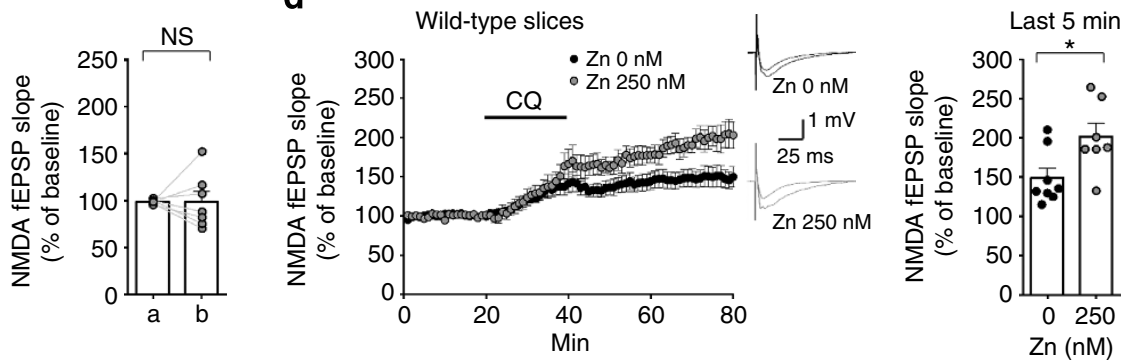

f

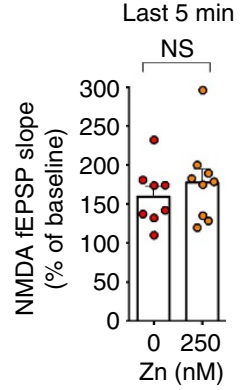

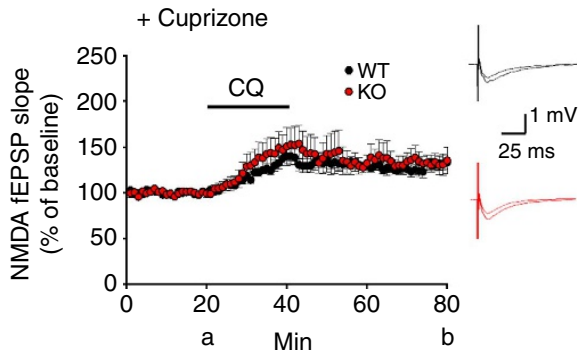

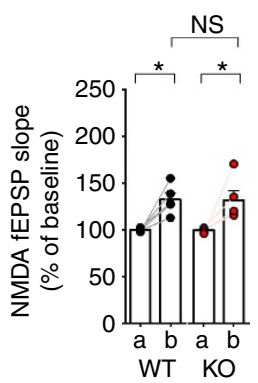

Figure 3 | CQ-dependent NMDAR activation requires $\mathbf{Z n}$ mobilization from pre- to postsynaptic sites. (a,b) CQ ( $4 \mu \mathrm{M}, 20 \mathrm{~min})$ fails to enhance NMDAR function at hippocampal SC-CA1 synapses in the presence of Ca-EDTA or TPEN (Zn chelators more potent than CQ), as measured by NMDAfEPSPs. Shank2-/- hippocampal slices were bath incubated with Ca-EDTA $(2 \mu \mathrm{M})$ or TPEN $(25 \mu \mathrm{M})$ throughout recordings. The labels a and $\mathrm{b}$ indicate 5 -min durations before CQ and the end of recordings, respectively. (Ca-EDTA, $n=6$ slices ( 3 animals) for WT and 6 (3) for KO; Student's t-test; TPEN, $n=6$ (2) for WT and 4 (2) for KO; Student's $t$-test). (c) CQ fails to enhance NMDAR function at $Z n T 3^{-/-}$hippocampal SC-CA1 synapses. ( $n=7$ slices (3 animals); Student's $t$-test). (d,e) Exogenously added Zn (250 nM) enhances NMDAR function at WT but not Shank2-/- synapses. Additional Zn was bath applied throughout the recording. ( $n=8$ slices (7 animals) for $0 \mathrm{nM}, 7$ (4) for $250 \mathrm{nM}$ in WT, and 8 (5) for $0 \mathrm{nM}, 9$ (4) for $250 \mathrm{nM}$ in KO; ${ }^{\star} P<0.05$; Student's t-test). (f) CQ enhances NMDAR function in the presence of cuprizone $(100 \mu \mathrm{M})$, a $\mathrm{Cu}^{2+}$-specific chelator. ( $n=5$ slices $(4$ animals) for WT and 5 (4) for $\mathrm{KO} ;{ }^{\star} P<0.05$; Student's $t$-test). Data in all panels with error bars represent mean \pm s.e.m. NS, not significant. 
disinhibition of NMDARs by CQ-mediated chelation of $\mathrm{Zn}$ in the synaptic cleft.

If the $\mathrm{Zn}$-ionophoric activity of CQ is the more likely candidate mediator of NMDAR activation, this raises the question: what is the source of $\mathrm{Zn}$ for NMDAR activation? One possible candidate is the $\mathrm{Zn}$ pool in presynaptic neurotransmitter vesicles, a major source of $\mathrm{Zn}$ in the brain that requires the $\mathrm{ZnT} 3$ transporter for its maintenance $\mathrm{e}^{21-24}$. In tests of this possibility using ZnT3deficient $\left(Z n T 3^{-/-}\right)$mice, we found that CQ had no effect on NMDAR activity in $Z n T 3^{-/}-$SC-CA1 synapses (Fig. 3c), suggesting that the presynaptic $\mathrm{Zn}$ pool is required for the CQ effect. In a control experiment, we confirmed that $\mathrm{Zn}$ signals are indeed largely absent in the $Z n T 3^{-/-}$hippocampus (Supplementary Fig. 3d).

The results described to this point were obtained in experiments performed without exogenous addition of $\mathrm{Zn}$ to brain slices, relying on the physiological concentrations of free $\mathrm{Zn}$ in the extracellular space. Previous studies have reported free $\mathrm{Zn}$ concentrations in the central nervous system of $\sim 20 \mathrm{nM}$ (ref. 56), although higher concentrations might be possible ${ }^{24}$. Here, we tested whether increasing the extracellular $\mathrm{Zn}$ concentration to $250 \mathrm{nM}$ affected CQ-dependent NMDAR activation. We found that $250 \mathrm{nM} \mathrm{Zn}$ had no significant effect on CQ-dependent NMDAR activation at Shank2-/- SC-CA1 synapses, as measured by NMDA-fEPSPs (Fig. 3d,e). This result suggests that additional $\mathrm{Zn}$ is not required for CQ-dependent NMDAR activation at Shank $2^{-/-}$synapses, implying that the presynaptic $\mathrm{Zn}$ pool under physiological conditions is sufficient for CQ-dependent NMDAR activation. Interestingly, $250 \mathrm{nM} \mathrm{Zn}$ caused a significant increase in NMDA-fEPSPs at WT synapses (Fig. 3d,e), a differential effect that warrants further investigation.

We next attempted to visualize CQ-dependent increases in $\mathrm{Zn}$ levels in postsynaptic compartments, using ZnAF-2DA, a membrane-permeable $\mathrm{Zn}$ indicator that, once inside the cell, is modified and trapped to indicate intracellular $\mathrm{Zn}$ levels ${ }^{57,58}$.
When WT mice were treated with CQ for $2 \mathrm{~h}$, Zn signals measured by two-photon confocal microscopy were significantly increased in both dendritic and cell body area of the hippocampal CA1 region, compared with vehicle-treated controls (Fig. 4a-f; and Supplementary Movies 1 and 2), consistent with the previous results obtained using a regular confocal microscope $e^{58}$.

Finally, because CQ can bind $\mathrm{Cu}^{2+}\left(K_{\mathrm{d}} \approx 10^{-8.9}\right)$ in addition to $\mathrm{Zn}$, we tested whether the $\mathrm{Cu}^{2+}$-binding activity of $\mathrm{CQ}$ also contributed to its effects on NMDAR function. Application of cuprizone, a selective $\mathrm{Cu}^{2}+$ chelator, to hippocampal slices before CQ treatment did not inhibit the CQ-induced increase in NMDAR activity (Fig. 3f), suggesting that the Cu-binding activity of CQ is not important for NMDAR activation. Taken together, these results suggest that CQ enhances NMDAR function through its $\mathrm{Zn}$-ionophoric, but not $\mathrm{Zn}$-chelating or $\mathrm{Cu}^{2+}$-binding, activity, and uses mainly the presynaptic $\mathrm{Zn}$ pool for NMDAR activation.

CQ activates NMDARs through postsynaptic Src. The results described thus far suggest that CQ mobilizes $\mathrm{Zn}$ from presynaptic vesicles into the synaptic cleft and postsynaptic compartments. $\mathrm{Zn}$ in the synaptic cleft is unlikely to contribute to NMDAR activation because $\mathrm{Zn}$ released presynaptically during neuronal activity is known to inhibit NMDARs ${ }^{24}$, and we demonstrated that Ca-EDTA has no effect on NMDA-fEPSPs (Supplementary Fig. 7a).

If postsynaptic $\mathrm{Zn}$ delivery is an important factor, then what would be the underlying mechanism for NMDAR activation? Previous studies have shown that $\mathrm{Zn}$ binds to and inactivates C-terminal Src kinase, a negative regulator of SFKs, which phosphorylate and activate NMDARs ${ }^{27,59}$. In related experiments, we found that two independent SFK inhibitors, PP2 and SU6656, applied to hippocampal slices before CQ treatment abolished CQ activation of NMDARs at both

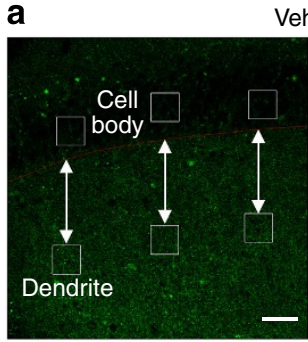

Slice depth $(5 \mu \mathrm{m})$

c

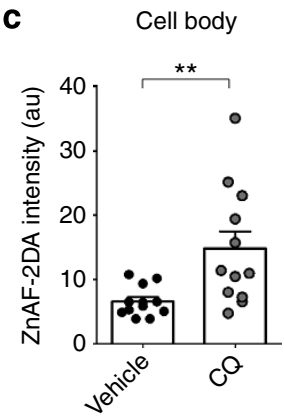

ehicle

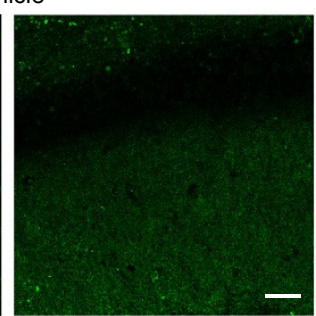

Slice depth $(50 \mu \mathrm{m})$

d

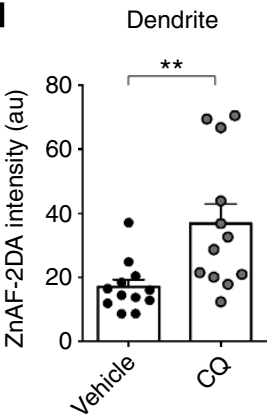

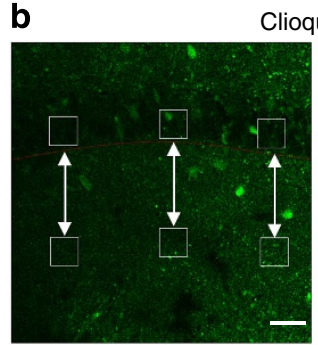

Slice depth $(5 \mu \mathrm{m})$

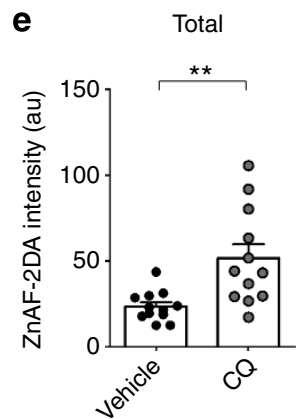

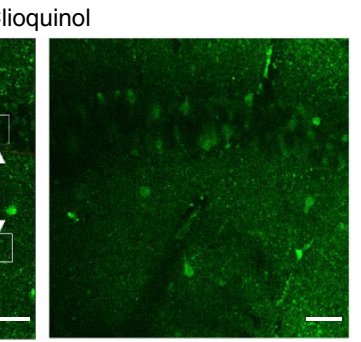

Slice depth $(50 \mu \mathrm{m})$

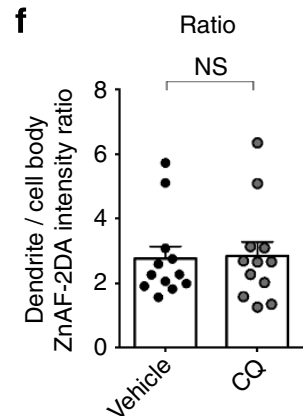

Figure 4 | CQ treatment increases $\mathbf{Z n}$ signals in the dendritic and cell body areas in the hippocampal CA1 region. (a-f) Mice (3-5 weeks) were injected with CQ (30 $\mathrm{mg} \mathrm{kg}^{-1}$; i.p), or vehicle, $2 \mathrm{~h}$ before brain slicing, and hippocampal slices (300 $\mu \mathrm{m}$ thick) were incubated with ZnAF-2DA (40 min) followed by two-photon microscopy. Zn signals (average intensities) were measured in the indicated three square regions in the cell body, or dendritic, area of the hippocampal CA1 region. Scale bars, $50 \mu \mathrm{m}(\mathbf{a}, \mathbf{b})$. $\left(n=12\right.$ slices ( 6 animals) for vehicle and 12 slices ( 5 animals) for $C Q ;{ }^{\star} P<0.05,{ }^{\star \star} P<0.01$, Student's $t$-test). Data in all panels with error bars represent mean \pm s.e.m. Au, arbitrary unit; NS, not significant. 
Shank $2^{-/-}$and WT SC-CA1 synapses (Fig. 5a,b). In control experiments, PP3, an inactive PP2 analogue, failed to block the NMDAR activation (Fig. 5c).

To further confirm that CQ enhances NMDAR function through SFKs and to test that the subcellular site of SFK action is indeed postsynaptic, we used the Src-inhibitory peptide $\operatorname{Src}(40-58)$, which contains a sequence corresponding to Src amino-acid residues $40-58$ and selectively blocks endogenous Src but not other SFK members ${ }^{59-61}$. Inclusion of this peptide $\left(0.03 \mathrm{mg} \mathrm{ml}^{-1}\right)$ in the patch pipette during patch-clamp recordings prevented CQ from increasing NMDAR activity at Shank $2^{-1-}$ and WT synapses, determined by measuring NMDA-eEPSCs (Fig. 5d,e). In control experiments, a scrambled Src-peptide variant (sSrc 40-58) had no effect on the CQ-dependent increase in NMDAR activity.

In contrast to NMDARs, AMPAR function was unaffected by the Src-inhibitory peptide, as determined from simultaneous recordings of AMPA-eEPSCs at both WT and Shank2-1- synapses (Fig. 5d,e). Consistent with this, the CQ-dependent increase in the NMDA/AMPA ratio determined from these currents was blocked by the Src-inhibitory peptide, but not by the scrambled peptide (Supplementary Fig. 8).

We also tested whether CQ treatment enhances Src activity, in addition to NMDAR function, by immunoblot analysis of CQ-treated hippocampal slices. We found that the levels of tyrosine phosphorylation of Src at Y416, known to render Src fully active ${ }^{59}$, were increased in CQ-treated WT and Shank $2^{-1-}$ slices, which was blocked by PP2 (Fig. 6a,b,d,e). In contrast, tyrosine phosphorylation of Src at Y527, known to stabilize the inactive conformation of $\mathrm{Src}^{59}$, was not affected by CQ treatment (Fig. 6a,c,d,f), suggesting that CQ promotes Src activation through the phosphorylation of distinct tyrosine residues.

Finally, in order to explore the involvement of other signalling pathways in the downstream of NMDAR activation, we tested inhibitors of MAPK kinase/MEK (PD98059) and CaMKII $\alpha$ (KN93). We found that suppression of MAPK/Erk by the
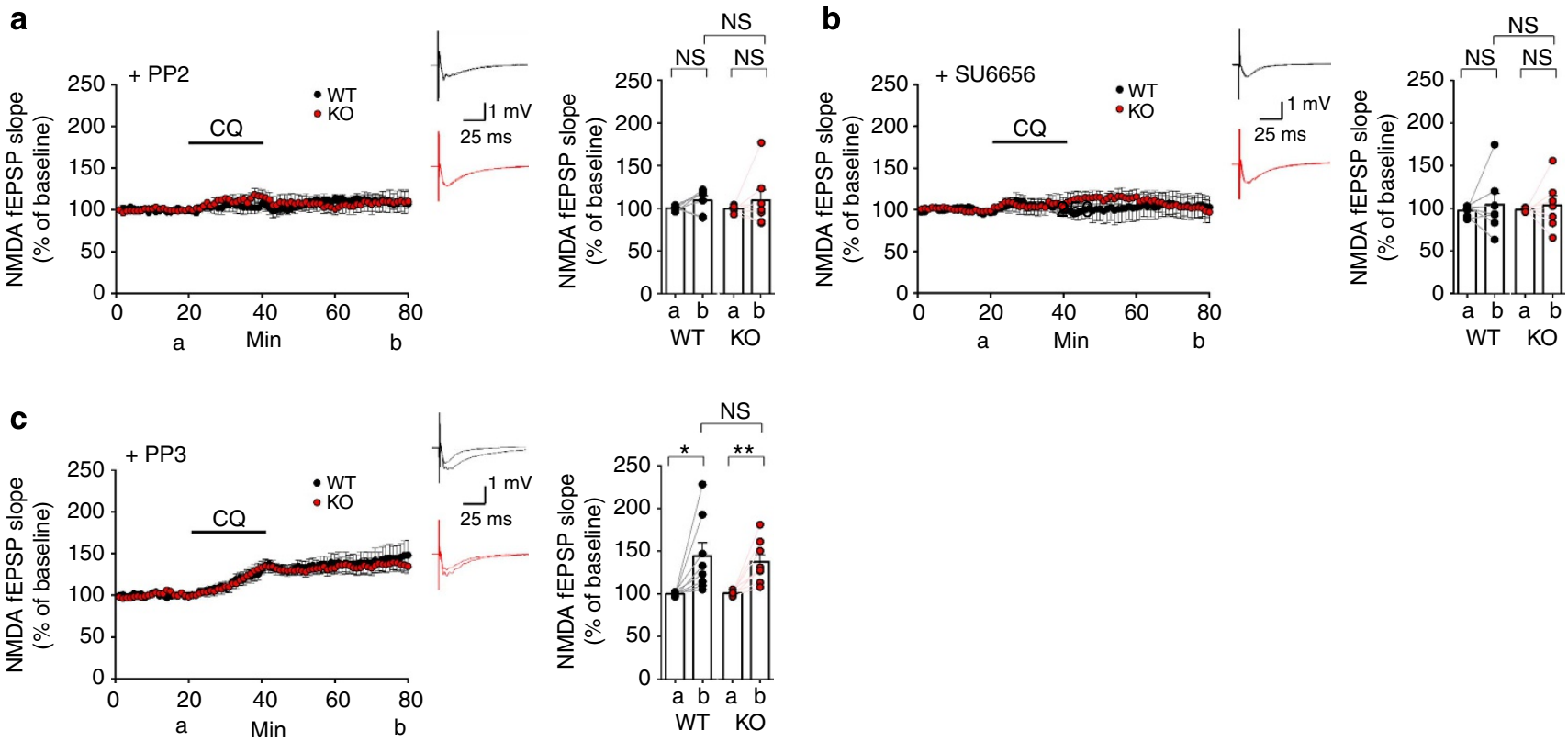

d
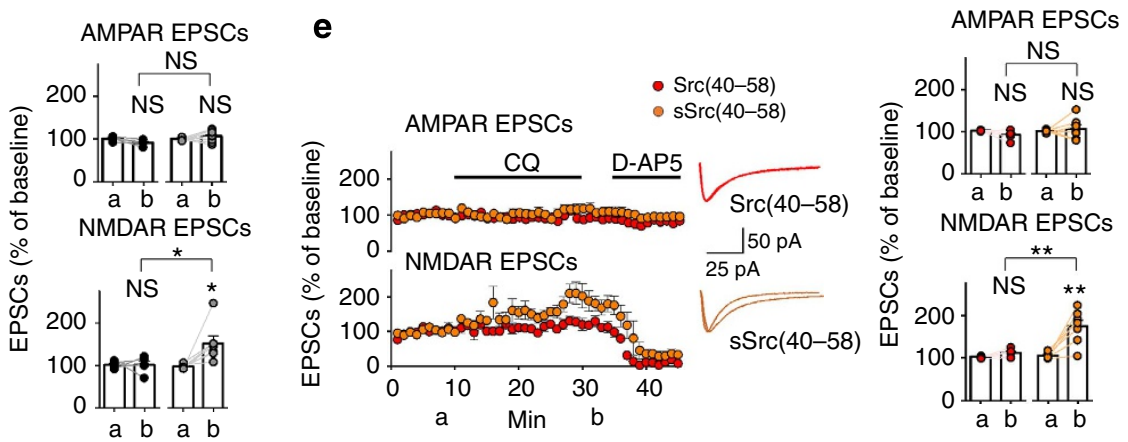

Figure 5 | CQ treatment activates NMDARs through postsynaptic Src activation. (a-c) CQ (4 $\mu$ M, 20 min) fails to enhance NMDAR function at Shank2 ${ }^{-/}$- SC-CA1 synapses in the presence of PP2 or SU6656, specific inhibitors of SFKs, but effectively enhances NMDAR function in the presence of PP3, an inactive PP2 analogue, as measured by NMDA-fEPSPs. Hippocampal slices were bath incubated with PP2 (10 $\mu$ M) or SU6656 (10 $\mu$ M) throughout recordings. (PP2, $n=7$ slices (6 animals) for WT and 7 (5) for KO; SU6656, $n=7$ (5) for WT and 7 (4) for KO; PP3, n= 8 (4) for WT and 8 (5) for KO; ${ }^{\star} P<0.05,{ }^{\star \star} P<0.01$; Student's $t$-test). (d,e) CQ fails to enhance NMDAR function at Shank2 ${ }^{-/}-$SC-CA1 synapses (e) in the presence of Src(40-58), a specific peptide inhibitor of Src, but effectively enhances NMDAR function in the presence of sSrc(40-58), a scrambled version of the peptide, as determined by simultaneous measurements of NMDA- and AMPA-eEPSCs at $-40 \mathrm{mV}$. NMDA-eEPSCs were measured at $60 \mathrm{~ms}$ after the stimulation. The labels $a$ and $b$ indicate 5 -min duration before and after $C Q$, respectively. (Src(40-58), $n=5$ cells ( 4 animals) for WT and 6 (4) for KO; sSrc(40-58), $n=7$ (5) for $\mathrm{WT}, 7$ (6) for $\mathrm{KO} ;{ }^{\star} P<0.05,{ }^{\star \star} P<0.01$; Student's $t$-test, compared with the 5-min duration before $\mathrm{CQ}$ ). Data in all panels with error bars represent mean \pm s.e.m. NS, not significant. 

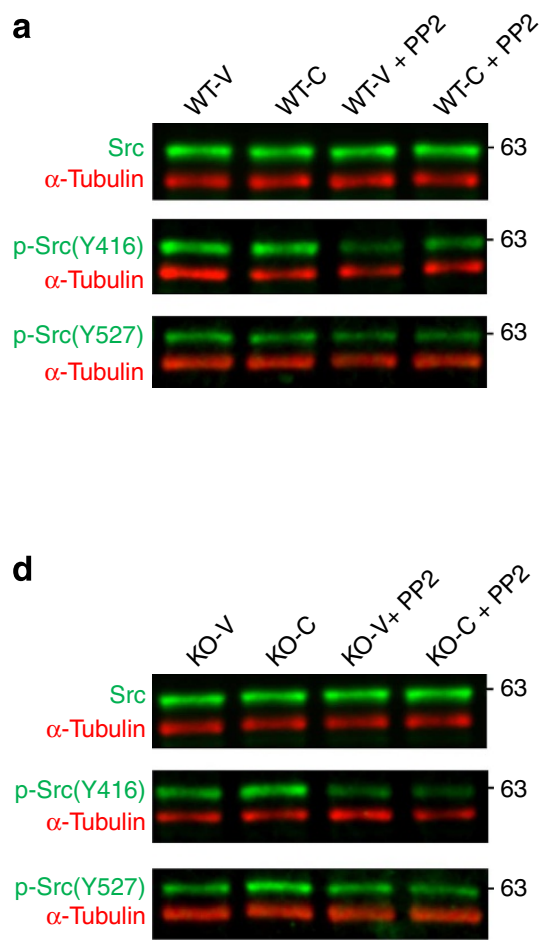

b

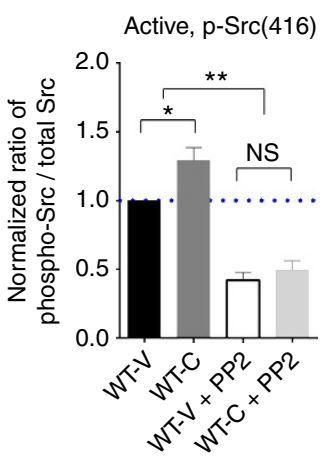

e

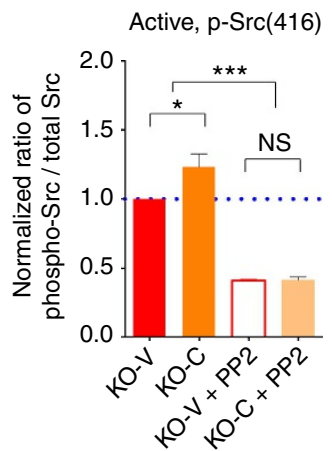

C

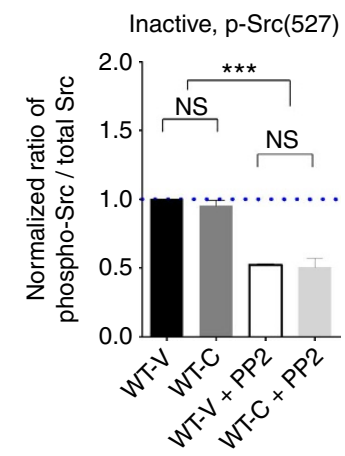

$\mathbf{f}$

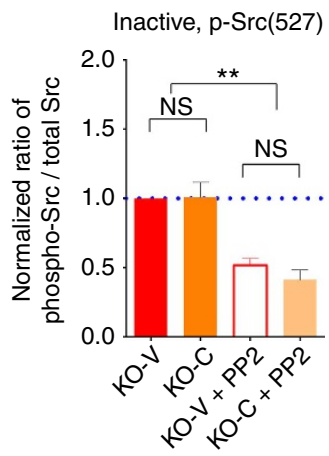

Figure 6 | CQ increases Src tyrosine phosphorylation in the hippocampus. (a-f) Treatment of WT (a-c) and Shank2-/- (d-f) hippocampal slices

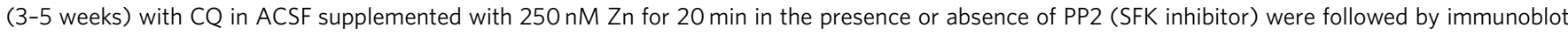
analysis of total and tyrosine phosphorylated Src (Y416 and Y527). For quantification, levels of Src tyrosine phosphorylation were normalized to total Src levels. $250 \mathrm{nM} \mathrm{Zn}$ was added to maximize the visualization of the changes occurring in Src tyrosine phosphorylation. ( $n=6$ slices ( 3 mice) for WT-V, WT-C, KO-V and KO-C; ${ }^{\star} P<0.05,{ }^{\star \star} P<0.01,{ }^{\star \star \star} P<0.001$; one-way analysis of variance with Tukey's post hoc analysis). Data in all panels with error bars represent mean \pm s.e.m. NS, not significant.

inhibition of MAPKK/MEK in the upstream had no effect on CQ-dependent NMDAR activation (Supplementary Fig. 9a). Intriguingly, CaMKII $\alpha$ inhibition caused a small reduction in the levels of CQ-induced NMDAR activation after but not during CQ treatment (Supplementary Fig. 9b), suggesting that CaMKII $\alpha$ is required for the maintenance of enhanced NMDAR function. These results collectively suggest that the CQ-induced increase in NMDAR function is dependent on Src, and imply that the subcellular site of Src activation is postsynaptic.

CQ treatment improves social interaction in $\mathrm{Tbrr}^{+/-}$mice. Finally, we considered whether CQ could rescue social interaction deficits in other mouse models of ASD in which reduced NMDAR is associated with autistic-like behaviours. One such model is the recently developed Tbr1-haploinsufficient $\left(\mathrm{Tbr} 1^{+/-}\right)$mouse, which has been reported to display a reduction in social interaction that is normalized by the NMDAR agonist $\mathrm{D}$-cycloserine ${ }^{50}$. We thus tested whether CQ could also rescue social interaction in these mice.

$T b r 1^{+/-}$mice showed reduced social interaction in the threechamber test, compared with WT mice, and acutely injected CQ $\left(30 \mathrm{mg} \mathrm{kg}^{-1}\right)$, administered $3 \mathrm{~h}$ before the three-chamber test, rescued the social interaction deficits of $T b r 1^{+/-}$mice, with no effect on WT mice, as determined by time spent in exploration/ sniffing, time spent in chamber and the preference index derived from exploration or chamber time (Fig. $7 \mathrm{a}-\mathrm{c}$ and Supplementary Fig. $10 \mathrm{a}-\mathrm{d}$ ). The positive rescue based on chamber time is supported by the strong correlation between exploration time and chamber time observed in $\mathrm{Tbr} 1^{+/-}$mice (Supplementary Fig. 11). CQ, however, did not affect social novelty recognition in $T b r 1^{+/-}$or WT mice, determined based on the preference for a new stranger mouse relative to a previously encountered stranger mouse (Fig. 7d-f and Supplementary Fig. 10e-h). These results suggest that CQ rescues social interaction deficits, but has no effect on social novelty recognition, in $T b r 1^{+/-}$mice. Taken together with similar results obtained in Shank2 $2^{-/-}$mice, this suggests that CQ is capable of rescuing social interaction deficits in two independent mouse models of ASD characterized by reduced NMDAR function.

CQ restores NMDAR function at $\mathrm{Tbr} \mathbf{1}^{+/-}$amygdalar synapses. We hypothesized that the CQ-dependent social rescue in $\mathrm{Tbr} 1^{+/-}$mice might be associated with the restoration of reduced NMDAR function at Tbr $1^{+/-}$synapses. We first examined synaptic transmission at $T b r 1^{+/}-$synapses in the hippocampus, where reduced NMDAR function was observed in Shank2 $2^{-/-}$ mice. However, no significant differences could be observed in the electrophysiological parameters, including miniature EPSCs (mEPSCs) in CA1 pyramidal neurons, and input-output ratio, paired-pulse ratio and NMDA/AMPA ratio at SC-CA1 synapses (Supplementary Fig. 12).

In contrast, principal neurons in the lateral amygdala (LA), a brain region also enriched with glutamate- and $\mathrm{Zn}$-releasing neurons ${ }^{62}$, showed slightly increased mEPSC amplitude but not frequency, without a change in the input-output ratio (Fig. 8a,b). Importantly, thalamic-LA Tbr $1^{+/-}$synapses showed a reduction 
a

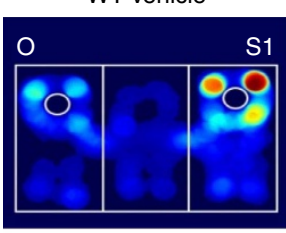

HT vehicle

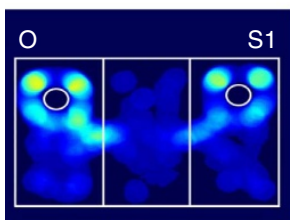

d WT vehicle

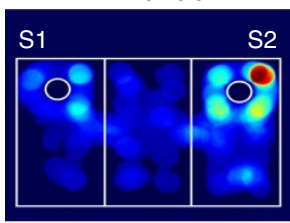

HT vehicle

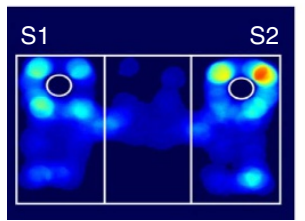

WT clioquinol

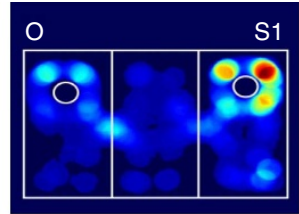

HT clioquinol

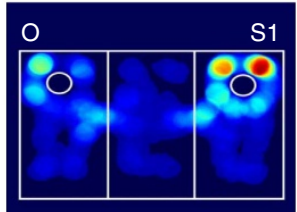

HT clioquinol

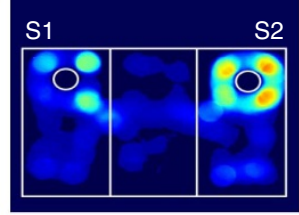

HT clioquinol

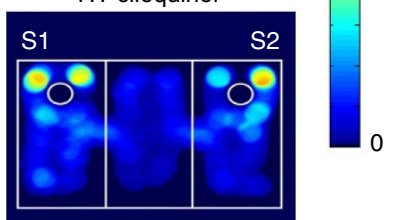

b

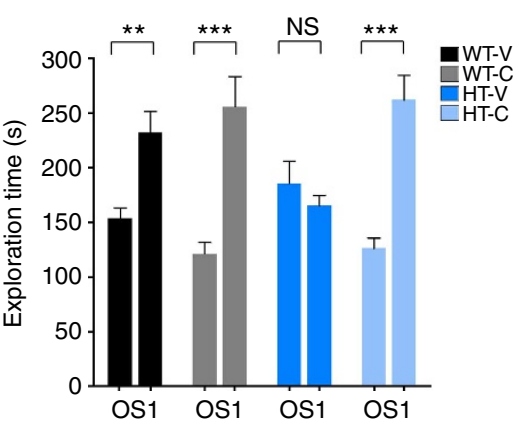

e

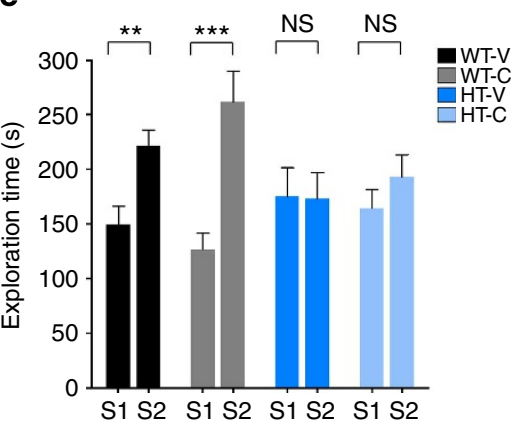

c

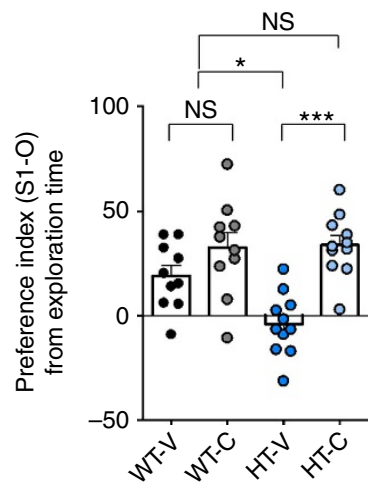

f

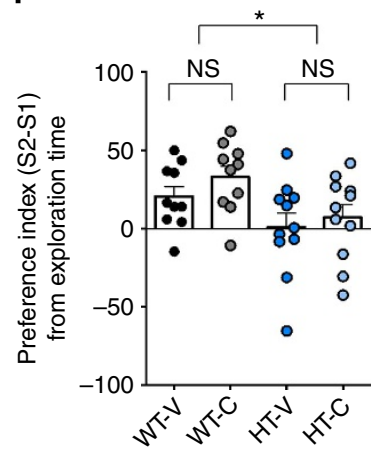

Figure 7 | CQ treatment improves social interaction in $\mathbf{T b r} \mathbf{1}^{+/-}$mice. (a-f) CQ improves social interaction (a-c) but not social novelty recognition (d-f) in $\mathrm{Tbr}^{+/-}(\mathrm{HT})$ mice, but has no effect in WT mice, as measured by the time spent in exploring targets and the social preference index derived from these results. Heat maps in a and $\mathbf{d}$ represent examples of mouse movements. ( $n=10$ for WT-V and WT-C, and 11 for $H T-V$ and $H T-C$; ${ }^{\star} P<0.05$, ${ }^{\star \star} P<0.01$, ${ }^{\star \star \star} P<0.001$; two-way analysis of variance (ANOVA) and one-way ANOVA with Tukey's post hoc test). Data in all panels with error bars represent mean \pm s.e.m. NS, not significant.

in the NMDA/AMPA ratio, which was normalized by CQ treatment (Fig. 8c,d). CQ did not cause a significant increase in the NMDA/AMPA ratio in WT mice. These results suggest that Tbr1 heterozygosity causes NMDAR hypofunction selectively in the amygdala, and its normalization is associated with the CQ-dependent social rescue in $T b r 1^{+/-}$mice.

\section{Discussion}

In the present study, we found that trans-synaptic $\mathrm{Zn}$ mobilization improves social interaction in two distinct mouse models of ASD through postsynaptic Src and NMDAR activation.

Our study suggests that CQ-dependent mobilization of $\mathrm{Zn}$ from pre- to postsynaptic sites-not $\mathrm{Zn}$ removal after chelationmight be useful in the treatment of ASDs. This unique transsynaptic $\mathrm{Zn}$ mobilization is supported by the following findings: (1) CQ failed to enhance NMDAR function in $Z n T 3^{-/-}$mice, which lack the presynaptic $\mathrm{Zn}$ pool; and (2) Ca-EDTA, a membrane-impermeable $\mathrm{Zn}$ chelator that should chelate $\mathrm{Zn}$ in the synaptic cleft or extracellular sites, blocked CQ-dependent NMDAR activation.

$\mathrm{CQ}$ can bind $\mathrm{Cu}$ and $\mathrm{Fe}$ in addition to $\mathrm{Zn}$. However, CQ appears to exert its effects through $\mathrm{Zn}$ interaction. A previous study has shown that $\mathrm{CQ}$ can mobilize $\mathrm{Zn}$ and $\mathrm{Cu}$ but not $\mathrm{Fe}$ into cytoplasmic sites in neuroblastoma cells ${ }^{63}$. In addition, our study shows that cuprizone, a specific $\mathrm{Cu}$ chelator, does not block CQ-dependent NMDAR activation. Regarding the potential involvement of the 'chelating' activity of CQ, as opposed to the 'ionophoric' activity, we suspect it is unlikely because CQ treatment of WT mice for $2 \mathrm{~h}$ did not lead to the reduction of $\mathrm{Zn}, \mathrm{Cu}$ or $\mathrm{Fe}$ in the brain. However, it should be pointed out that the proposed $\mathrm{Zn}$ mobilization by CQ should involve $\mathrm{Zn}$ chelation at presynaptic sites before $\mathrm{Zn}$ ions are mobilized to postsynaptic sites through ionophoric effects.

We propose a specific mechanism that may underlie the CQ-dependent social rescue, namely NMDAR activation through postsynaptic Src. In support of this, CQ-dependent NMDAR activation at Shank2 $2^{-/}$hippocampal synapses is blocked by two independent inhibitors of SFKs (PP2 and SU6656), as well as the Src-inhibitory peptide $\operatorname{Src}(40-58)$, which acts in the postsynaptic compartments when applied through patch pipettes. In addition, CQ treatment increases Src phosphorylation at Y416 but not Y527. We initially expected that the phosphorylation at Src Y527, which keeps Src at an inactive conformation, may be reduced to activate Src because Y527 is the substrate of $\mathrm{Zn}$ inhibited C-terminal Src kinase ${ }^{59}$. On the contrary, we found an increase in the phosphorylation of Src Y416, which is known to render Src fully active. Although further details remain to be studied, CQ appears to promote full activation of Src after its initial activation by some other mechanisms.

Our study does not exclude the possibility that postsynaptic $\mathrm{Zn}$ acts on targets other than SFKs and NMDARs. For instance, a previous study has shown that $\mathrm{Zn}$ enhances excitatory synaptic stabilization of Shank $2 / 3$ and synapse formation and maturation $^{30}$. Because two independent inhibitors of SFKs and the Src-inhibitory peptide significantly blocked the 
a

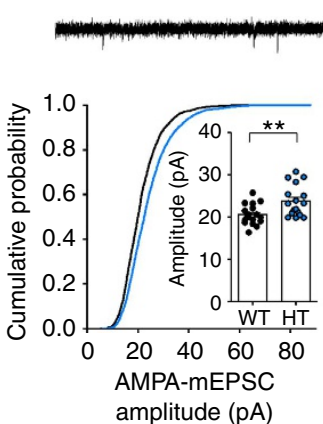

HT

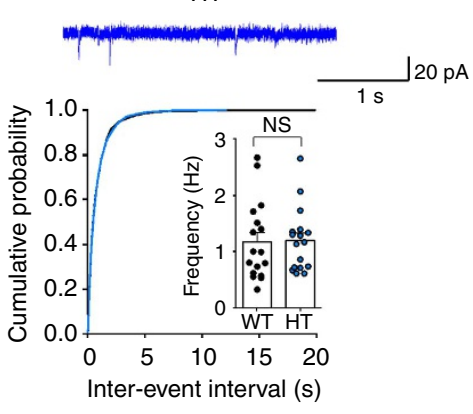

b

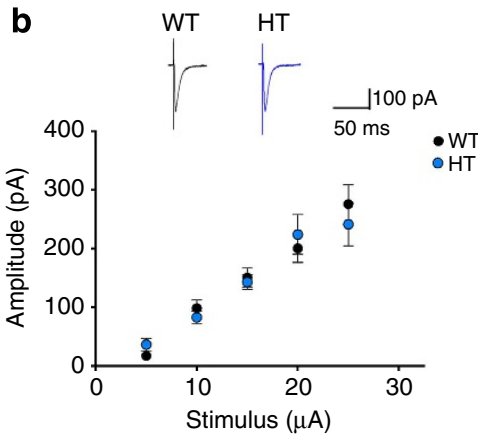

C

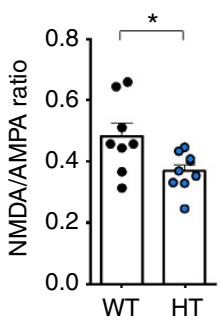

WT

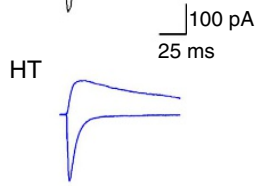

d

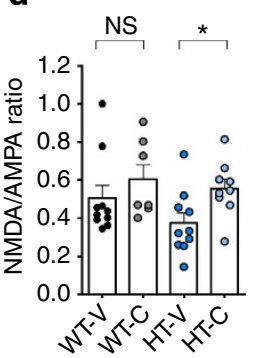

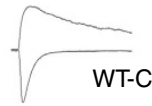

WT-C

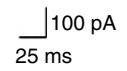

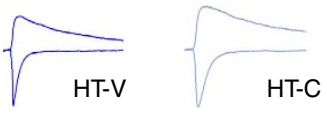

Figure 8 | CQ restores NMDAR function at $\mathbf{T b r 1}^{+/-}$amygdalar thalamic-LA (T-LA) synapses. (a) CQ increases mEPSC amplitude but not frequency in $\mathrm{Tbr}^{+/}{ }^{+}$- principal neurons in the LA ( $4-6$ weeks). ( $n=17$ cells ( 3 animals) for WT and HT, ${ }^{\star \star} P<0.01$, Student's $t$-test). (b) CQ has no effect on the inputoutput ratio at $\mathrm{Tbr} \mathrm{1}^{+/}-\mathrm{T}$-LA synapses (4-6 weeks), as indicated by plots of fEPSP slopes against stimulus intensities. Representative current traces are an average of three consecutive responses with input stimulations of $25 \mu \mathrm{A}$. ( $n=8$ cells ( 3 animals) for WT and HT; Student's t-test). (c) Reduced NMDA/ AMPA ratio at $\mathrm{Tbr}{ }^{+/} /-$T-LA synapses ( $4-6$ weeks). ( $n=8$ cells (5 animals) for WT and 9 (5) for HT; ${ }^{\star} P<0.05$; Student's $t$-test). (d) CQ restores the NMDA/AMPA ratio at Tbr $1^{+/-}$T-LA synapses but has no effect on WT synapses (4-6 weeks). ( $n=10$ (5) for WT-V, 7 (4) for WT-C, 10 (6) for HT-V and 9 (4) for HT-C; ${ }^{\star} P<0.05$; two-way analysis of variance (ANOVA) and one-way ANOVA with Tukey's post hoc test). Data in all panels with error bars represent mean \pm s.e.m. NS, not significant.

CQ-dependent NMDAR activation (Fig. 5), we did not explore this possibility. It should be noted, however, that the increase in CQ-induced NMDA-fEPSPs caused by additional $\mathrm{Zn}(250 \mathrm{nM})$ was greater at WT synapses than at Shank2-/- synapses (Fig. 3d,e). This suggests that postsynaptic Shank2 may contribute to Zn-dependent NMDAR activation during high levels of neuronal activity and thus may also contribute to the aetiology of ASDs.

Previous studies have reported the roles of CQ in the regulation of synaptic transmission, including those from Dr Takeda's group $^{57,58}$. The latter studies suggest that an increase in intracellular $\mathrm{Zn}$ concentration in the postsynaptic side of CA1 synapses inhibits NMDAR-dependent LTP, which apparently differ from our results that CQ has no effect on LTP at WT SC-CA1 synapses, and that CQ enhances LTP at Shank2-/SC-CA1 synapses (Fig. 2b). However, because Shank2-/synapses display substantial alterations in synaptic protein composition/modification and synaptic transmission/plasticity ${ }^{45}$, the CQ-dependent NMDAR activation at Shank2-/- synapses cannot be compared with the previous results. Regarding the effect of CQ on LTP at WT synapses, the difference might stem from that (1) previous studies used rat hippocampal slices, whereas we used mouse ones, and (2) previous studies prepared brain slices $2 \mathrm{~h}$ after i.p. injection of $\mathrm{Zn}-\mathrm{CQ}\left(9.8 \mu \mathrm{mol}(\sim 3 \mathrm{mg}) \mathrm{kg}^{-1}\right)$ and measured LTP without CQ in the artificial CSF (ACSF), whereas we introduced CQ $(4 \mu \mathrm{M})$ directly to slices obtained from CQuntreated mice during electrophysiological measurements. Notably, an independent paper has also reported that bath application of CQ $(4 \mu \mathrm{M})$ has no effect on LTP at SC-CA1 synapses in the mouse brain, similar to our data ${ }^{63}$. To address this issue directly, we mimicked the method of CQ treatment reported in Dr Takeda's group, preparing mouse hippocampal slices $2 \mathrm{~h}$ after i.p. injection of $\mathrm{Zn}-\mathrm{CQ}\left(9.8 \mu \mathrm{mol} \mathrm{kg}^{-1}\right)$. However, we found no difference between treated and untreated groups in LTP induced by high-frequency stimulation at SC-CA1 synapses $(n=7$ slices, 4 mice for vehicle and 7, 5 for CQ; data not shown). Therefore, the differences may be attributable to the fact that different animal species were used.

CQ-dependent rescue of social deficits in Shank2-/- and Tbr $1^{+/-}$mice was associated with CQ-dependent elevation of NMDAR function, further supporting the hypothesis that NMDAR hypofunction may underlie ASDs. This concept was put forward based on the observations that D-cycloserine improves ASD symptoms in humans and autistic-like phenotypes in animals (reviewed in ref. 64), although further studies are needed to verify this hypothesis.

CQ rescues social interaction in Shank $2^{-/-}$and $T b r 1^{+/-}$ mice, but it fails to rescue social novelty recognition, repetitive behaviour, hyperactivity or anxiety-like behaviour in Shank $2^{-/-}$ mice. This is reminiscent of the previous result that NMDAR activation by $\mathrm{D}$-cycloserine selectively rescues social interaction in Shank $2^{-/-}$mice $^{45}$. This selective rescue might be attributable to the different nature of the circuits associated with these behaviours, where some are reversible, or at least treatable, whereas others are not. In line with this, NMDARs are involved in the regulation of both neuronal development and synaptic transmission/plasticity/signalling ${ }^{11,65}$. In addition, activitydependent sculpting of neuronal circuits associated with ASDs has critical time windows ${ }^{66}$.

Finally, our study broadens the therapeutic potential of CQ. CQ has been used as a topical antiseptic or an oral intestinal amoebicide since 1930s, although the latter use has ceased for its controversial association with subacute myelo-optic neuropathy ${ }^{52}$. Recently, however, CQ-dependent chelation of $\mathrm{Zn}$ has been 
suggested for the treatment of neurological disorders including Alzheimer's disease ${ }^{67}$, Parkinson's disease ${ }^{68}$ and Huntingtons' disease ${ }^{69}$. Moreover, PBT2, a second-generation CQ-related compound under clinical trials, seems to be safe and improve cognitive deficits in patients with Alzheimer's disease ${ }^{67}$. Therefore, our study is the first to demonstrate the possibility of repositioning of the FDA-approved antibiotic, CQ, to ASDs based on a novel mechanism distinct from chelation. In addition, CQ-dependent trans-synaptic $\mathrm{Zn}$ mobilization might also be useful in other psychiatric disorders that are notable for being caused by a decrease in NMDAR function ${ }^{70}$.

In conclusion, our study suggests that trans-synaptic $\mathrm{Zn}$ mobilization rapidly improves social interaction in two independent mouse models of ASD through Src and NMDAR activation, and a new therapeutic potential of CQ in the treatment of ASDs.

\section{Methods}

Mice. Shank $2^{-/-}$mice and $T b r 1^{+/-}$mice have been reported ${ }^{45,50}$. All mice were backcrossed to a C57BL/6 background for more than 20 generations, and housed and bred in a mouse vivarium at the Korea Advanced Institute of Science and Technology (KAIST; Shank2 $2^{-/-}$mice) and the Academia Sinica (Tbr1 $1^{+/-}$ mice). For breeding of Shank 2 mice, we used a scheme of heterozygous $(\mathrm{HT}) \times \mathrm{HT}$ to produce littermate pairs of WT and KO mice. To breed Tbrl mice, we used offspring from HT males $\times$ WT females. Other combinations did not yield any differences in breeding efficiency or behavioural phenotypes. Pups were kept with the dam until weaning at postnatal day 21 . After weaning, animals were housed in mixed-genotype groups of 3-5 mice per cages, and randomly subjected to electrophysiological and behavioural experiments. Animals at 3-5 weeks of age were used for electrophysiological experiments and two-photon imaging; male animals at 2-4 months of age were used for behavioural assays. For TFL-Zn staining, male animals at 8 weeks (for Shank $2^{-/-}$mice) were used. WT littermates were used as controls.

$\mathrm{ZnT3}^{-/-}$mice, reported previously ${ }^{54}$, were maintained in the KAIST animal facility. These mice also were backcrossed to the C57BL/6 background for more than 10 generations. Both male and female animals at 3-5 weeks were used for electrophysiological experiments and TFL-Zn staining (P23).

All mice were bred and maintained according to the KAIST and Academia Sinica Animal Research Requirements, and all procedures were approved by the Committees of Animal Research at KAIST, and at Academia Sinica. Mice were fed ad libitum by standard rodent chow and tap water, and housed under $12 \mathrm{~h}$ light/dark cycle (lights off at 1900 hours in KAIST and at 2000 hours in Academia Sinica).

Clioquinol. CQ (Calbiochem) was dissolved in dimethylsulfoxide (DMSO; Sigma) and polyethylene glycol (Aldrich; DMSO:polyethylene glycol=1:9) to a final concentration of $20 \mathrm{gl}^{-1}$. WT and Shank2 $2^{-}-$mice (or $\mathrm{Tbr} 1^{+1-}$ mice) received acute i.p. injection of CQ $\left(30 \mathrm{mg} \mathrm{kg}^{-1}\right)$ or the same volume of DMSO-polyethylene glycol mixture. The injection was performed $2 \mathrm{~h}$ before (Shank $2^{-/-}$mice and WT littermates) or $3 \mathrm{~h}$ before ( $\mathrm{Tbr} 1^{+7}-$ mice and WT littermates) behavioural assays at the discretion of the facility.

Drug treatment scheme. We devised a within-subjects design with a 1-week washout period (Supplementary Fig. 1a), and divided animals into two groups, vehiclefirst and CQ-first group, to rule out carryover effects. Each mouse received a single acute dose of vehicle or CQ, and underwent a single behavioural task, one task per week. Testing was conducted in dedicated behavioural test rooms during the light phase (three-chamber test) and the dark phase (repetitive behaviours and open-field test).

Three-chamber social interaction assay. The three-chamber social interaction assay for Shank2 mice (Shank $2^{-/-}$and WT littermates) and Tbr1 mice ( $\mathrm{Tbr} 1^{+/}$ and WT littermates) were performed ${ }^{45,50}$. In short, the assay consisted of three phases of 10 min duration: habituation, social interaction (stranger 1 versus object) and social novelty recognition (stranger 1 versus stranger 2). Exploration was defined as instances in which WT or mutant mouse tries to sniff object/stranger, or orients its nose towards and come close to object/stranger. Individual movement tracks were analysed by Ethovision 10.0 (Noldus) and modified by customdesigned software MatLab (MathWorks) to generate heat maps. Time spent in exploration was analysed by the researcher who was blinded to the subject genotype (in Shank2 mice), or by using the Smart Video Tracking System (Panlab, in Tbr1 mice). In addition to exploration time, we used the preference index, which represents a numerical difference between time spent exploring the targets (stranger 1 versus object or stranger 2 versus stranger 1 ) divided by total time spent exploring both targets ${ }^{45}$.

Repetitive behaviours. Shank $2^{-/-}$mice and their WT littermates in their home cages without bedding were used to measure times spent in repetitive behaviours, including jumping and grooming during $10 \mathrm{~min}$. Jumping was defined as the behaviour of a mouse where it rears on its hind legs at the corner of the cage, or along the side walls, and jumps so that the two hind legs are simultaneously off the ground. Grooming behaviour was defined as stroking or scratching of face, head or body with the two forelimbs, or licking body parts ${ }^{45}$. The experiments and analyses were performed independently in a blind manner.

Open-field test. The size of the open-field box was $40 \times 40 \times 40 \mathrm{~cm}$, and the centre zone line was $13.3 \mathrm{~cm}$ apart from the edge. Mice were placed in the centre in the beginning of the test, and mouse movements were recorded with a video camera for $60 \mathrm{~min}$, and were analysed by Ethovision 10.0 (Noldus).

Electrophysiology. For hippocampal electrophysiological experiments, sagittal hippocampal slices $(400 \mu \mathrm{m}$ thick for extracellular and $300 \mu \mathrm{m}$ thick for intracellular recordings) of the mutant mice (Shank $2^{-/-}$mice, $T b r 1^{+/-}$mice or $\mathrm{ZnT3}^{-/-}$mice) and their WT littermates were prepared using a vibratome (Leica VT1200) in ice-cold dissection buffer containing (in $\mathrm{mM}$ ) 212 sucrose, $25 \mathrm{NaHCO}_{3}, 5 \mathrm{KCl}, 1.25 \mathrm{NaH}_{2} \mathrm{PO}_{4}, 0.5 \mathrm{CaCl}_{2}, 3.5 \mathrm{MgSO}_{4}, 10$ D-glucose, $1.25 \mathrm{~L}$-ascorbic acid and $2 \mathrm{Na}$-pyruvate bubbled with $95 \% \mathrm{O}_{2} / 5 \% \mathrm{CO}_{2}$. CA3 was removed to prevent epileptiform activity. For amygdalar electrophysiological experiments, coronal slices $(300 \mu \mathrm{m})$ including the LA of $\mathrm{Tbr} 1^{+7-}$ mice and their WT littermates were cut. The slices were recovered at $32^{\circ} \mathrm{C}$ for $1 \mathrm{~h}$ in normal ACSF (in mM: $124 \mathrm{NaCl}, 2.5 \mathrm{KCl}, 1 \mathrm{NaH}_{2} \mathrm{PO}_{4}, 25 \mathrm{NaHCO}_{3}, 10$ glucose, $2 \mathrm{CaCl}_{2}$ and $2 \mathrm{MgSO}_{4}$ oxygenated with $95 \% \mathrm{O}_{2} / 5 \% \mathrm{CO}_{2}$ ). For the recording, a single slice was moved to and maintained in submerged-type chamber at $28{ }^{\circ} \mathrm{C}$, continuously perfused with ACSF $\left(2 \mathrm{ml} \mathrm{min}^{-1}\right)$ saturated with $95 \% \mathrm{O}_{2} / 5 \% \mathrm{CO}_{2}$. Stimulation and recording pipettes were pulled from borosilicate glass capillaries (Harvard Apparatus) using a micropipette electrode puller (Narishege).

For extracellular recordings, mouse hippocampal slices at the age of postnatal day 21-35 were used. fEPSPs were recorded in the stratum radiatum of the hippocampal CA1 region using pipettes filled with ACSF (1 M $\Omega$ ). fEPSP was amplified (Multiclamp 700B, Molecular Devices) and digitized (Digidata 1440A, Molecular Devices) for measurements. The Schaffer collateral pathway was stimulated every $20 \mathrm{~s}$ with pipettes filled with ACSF $(0.3-0.5 \mathrm{M} \Omega)$. The stimulation intensity was adjusted to yield a half-maximal response, and three successive responses were averaged and expressed relative to the normalized baseline. To induce LTP, highfrequency stimulation $(100 \mathrm{~Hz}, 1 \mathrm{~s})$ was applied after a stable baseline was acquired. CQ $(4 \mu \mathrm{M})$ was bath applied before and after LTP induction during the whole experimental processes. To isolate NMDAR-mediated fEPSPs, we used ACSF containing $2 \mathrm{mM}$ calcium, $0.1 \mathrm{mM}$ magnesium and 6,7-dinitroquinoxaline-2,3-dione (10 $\mu \mathrm{M}, \mathrm{DNQX}$, Tocris), which inhibits AMPAR-mediated EPSPs.

Whole-cell patch-clamp recordings of hippocampal CA1 pyramidal neurons, and of LA principal neurons in the dorsolateral division were made using a MultiClamp 700B amplifier (Molecular Devices) and Digidata 1440A (Molecular Devices). During whole-cell patch-clamp recordings, series resistance was monitored each sweep by measuring the peak amplitude of the capacitance currents in response to short hyperpolarizing step pulse $(5 \mathrm{mV}, 40 \mathrm{~ms})$; only cells with a change in $<20 \%$ were included in the analysis. For afferent stimulation of hippocampal pyramidal neurons, the Schaffer collateral pathway was selected, while for that of LA, the thalamic afferent pathway was stimulated. For LA electrophysiology, brain slices were selected based on the presence of a wellisolated, sharply defined trunk (containing thalamic afferents) crossing the dorsolateral division of the LA, which is a site of convergence of somatosensory and auditory inputs. For NMDA/AMPA ratio experiments, mouse hippocampal slices (P17-P25) and LA slices (4-6 weeks old) were used. The recording pipettes (2.5-3.5 M $\Omega$ ) were filled with an internal solution containing the following (in mM): $100 \mathrm{CsMeSO}_{4}, 10$ TEA-Cl, $8 \mathrm{NaCl}, 10$ HEPES, 5 QX-314-Cl, $2 \mathrm{Mg}$-ATP, 0.3 Na-GTP and 10 EGTA, with pH 7.25, 295 mOsm). CA1 pyramidal neurons and LA principal neurons were voltage clamped at $-70 \mathrm{mV}$, and EPSCs were evoked at every $15 \mathrm{~s}$. AMPAR-mediated EPSCs were recorded at $-70 \mathrm{mV}$, and 30 consecutive responses were recorded after stable baseline. After recording AMPARmediated EPSCs, holding potential was changed to $+40 \mathrm{mV}$ to record NMDAR mediated EPSCs. NMDA component was measured at $60 \mathrm{~ms}$ after the stimulation. The NMDA/AMPA ratio was determined by dividing the mean value of 30 NMDA components of EPSCs by the mean value of 30 AMPAR-mediated EPSC peak amplitudes. Somatic whole-cell recording of mEPSCs were obtained in amygdalar principal neurons at a holding potential of $-70 \mathrm{mV}$. TTX $(1 \mu \mathrm{M})$ and picrotoxin $(100 \mu \mathrm{M})$ were added to ACSF to inhibit spontaneous action potential-mediated synaptic currents and IPSCs, respectively. CQ $(4 \mu \mathrm{M})$ was bath applied from $20 \mathrm{~min}$ before and during the whole period of NMDA/AMPA ratio recording. For measuring AMPAR-mediated and NMDAR-mediated EPSCs together upon CQ treatment, pyramidal neurons were voltage clamped at $-40 \mathrm{mV}$, and EPSCs were evoked at every $15 \mathrm{~s}$. AMPAR-mediated EPSC was determined as a peak amplitude of EPSC, and NMDAR-mediated EPSC as a component at $60 \mathrm{~ms}$ after stimulation. NMDA/AMPA ratio at $-40 \mathrm{mV}$ was calculated by using both values, and monitored during the experimental process. Src-inhibiting peptide, $\operatorname{Src}(40-58)$, and its analogue, scrambled $\operatorname{Src}(40-58$; ref. 61), were purchased from Peptron, and introduced into the internal solution at a concentration of $0.03 \mathrm{mg} \mathrm{ml}^{-1}$ to observe whether Src-inhibition affects the action of CQ. Picrotoxin $(100 \mu \mathrm{M})$ was always added to ACSF to block GABAA receptor-mediated currents. 
Data were acquired by Clampex 10.2 (Molecular Devices) and analysed by Clampfit 10 (Molecular Devices). Drugs were purchased from Tocris (DNQX, TPEN, PP2, PP3 and D-AP5), Abcam (PD98059) and Sigma (KN93, picrotoxin, Ca-EDTA, cuprizone and SU6656).

ZnAF-2DA imaging. For intracellular Zn staining, two groups of WT mice (3-5 weeks) were injected with vehicle (DMSO) or CQ $\left(30 \mathrm{mg} \mathrm{kg}^{-1}\right) 2 \mathrm{~h}$ before imaging. For two-photon imaging, sagittal hippocampal slices $(300 \mu \mathrm{m}$ thick) were immersed in $10 \mu \mathrm{M}$ ZnAF-2DA (Enzo Life Sciences) in ACSF for $40 \mathrm{~min}$, followed by $1 \mathrm{~h}$ washout with ACSF. Zn signals were measured by using a multiphoton laser scanning microscope system LSM 7 MP (Carl Zeiss). A total of $\sim 200 \mathrm{Z}$ stack images $(0.7 \mu \mathrm{m}$ interval; $\sim 130-180 \mu \mathrm{m}$ total depth) were captured from each slice. For quantification of $\mathrm{Zn}$ signals, a total of 11 images at every $4.5 \mu \mathrm{m}$ depth from the surface (from $\sim 5$ to $50 \mu \mathrm{m}$ depth) were selected. Three regions of interest (ROIs; squares of $33 \mu \mathrm{m}^{2}$ ) in the CA1 cell body area, or in the CA1 dendritic field, were analysed of $\mathrm{Zn}$ fluorescence signals using MetaMorph (Molecular Devices).

TFL-Zn staining. Without fixation, brain sections $(10 \mu \mathrm{m}$ thick) were stained with the $\mathrm{Zn}$-specific dye TFL-Zn ( $N$-(6-methoxy-8-quinolyl)-p-carbox-

ybenzoylsulfonamide $(0.1 \mathrm{mM})$, Calbiochem) dissolved in phosphate-buffered saline $(\mathrm{pH}$ 7.2), and photographed with a digital camera linked to a fluorescence microscope (Olympus IX71; excitation, 330-385 nm; dichromatic, $400 \mathrm{~nm}$; and barrier, $420 \mathrm{~nm}$ ). Fluorescence signals were obtained using an image programme (Image-Pro Insight, Media Cybernetics, Silver Spring, MD). Images of five consecutive hippocampal slices from an individual brain were quantified using MetaMorph (Molecular Devices). ROIs were defined as three to five squares $\left(50 \times 50 \mu \mathrm{m}^{2}\right)$ in WT and Shank $2^{-/-}$hippocampal DG, CA3 and CA1 regions (5, 3 and 5 squares, respectively). For quantification, the total fluorescence from a Shank2 ${ }^{-1}$ ROI was normalized to that from an equivalent WT ROI.

Src immunoblot analysis. For immunoblotting of Src proteins, WT and Shank $2^{-/-}$sagittal hippocampal slices $(400 \mu \mathrm{m}$ thick; $3-5$ weeks) were prepared using a vibratome (Leica VT1200). After recovery at $37^{\circ} \mathrm{C}$ for $1 \mathrm{~h}$ in normal ACSF supplemented with $250 \mathrm{nM} \mathrm{ZnCl}_{2}$, slices were treated with $\mathrm{CQ}$, or vehicle (DMSO), for $20 \mathrm{~min}$, in the presence or absence of PP2. After treatments, the slices were homogenized in $100 \mu$ lice-cold homogenization buffer $(0.32 \mathrm{M}$ sucrose, $10 \mathrm{mM}$ HEPES, pH 7.4, $2 \mathrm{mM}$ EDTA, protease inhibitors and phosphatase inhibitors) per each slice, and subjected to immunoblot analysis and quantification using Odyssey Fc Imaging System (LI-COR). The following antibodies were purchased: Src, phosphor-Src (Tyr-416; 1:1,000 dilution) and phosphor-Src (Tyr-527; Cell Signaling; 1:1,000 dilution). Full-size immunoblot images for Fig. 6 are shown in Supplementary Fig. 13.

Crude synaptosomes. Crude synaptosomes from Shank2 $2^{-/-}$mice were prepared as described ${ }^{45}$. Briefly, mouse brains ( 2 months old) were homogenized in ice-cold homogenization buffer $(0.32 \mathrm{M}$ sucrose, $10 \mathrm{mM}$ HEPES, $\mathrm{pH} 7.4,2 \mathrm{mM}$ EDTA, protease inhibitors and phosphatase inhibitors). The homogenates were centrifuged at $900 \mathrm{~g}$ for $10 \mathrm{~min}$. The resulting supernatant was centrifuged again at $12,000 \mathrm{~g}$ for $15 \mathrm{~min}$. The pellet was resuspended in homogenization buffer and centrifuged at $13,000 \mathrm{~g}$ for $15 \mathrm{~min}$ (the resulting pellet is P2; crude synaptosomes). This sample was immunoblotted with ZnT3 antibodies (SYSY).

Metal analysis. WT and Shank2 $2^{-/-}$mice (2-3 months) were treated with CQ $\left(30 \mathrm{~m} \mathrm{~kg}^{-1}\right)$, or DMSO, by i.p. injection $2 \mathrm{~h}$ before brain preparation and subsequent whole-brain metal analysis by inductively coupled plasma mass spectrometry.

Statistical analysis. We randomly performed all the behaviour experiments, ZnAF-2DA and TFL-Zn imaging, and metal analysis by researchers blind to the identity of the animals, and analysed the data in a blind manner. Data collection and analysis for slice electrophysiology and immunoblotting were performed randomly, but not blind to the conditions of the experiments. Statistical analyses were performed using Prism GraphPad (version 6.05) software, and details of the results are described Supplementary Data 1. No statistical methods were used to predetermine sample sizes, but our sample sizes are similar to those reported in previous related publications ${ }^{45,50}$. An outlier was defined as a value outside the mean \pm 3 s.d.

\section{References}

1. Huguet, G., Ey, E. \& Bourgeron, T. The genetic landscapes of autism spectrum disorders. Annu. Rev. Genomics Hum. Genet. 14, 191-213 (2013).

2. Jeste, S. S. \& Geschwind, D. H. Disentangling the heterogeneity of autism spectrum disorder through genetic findings. Nat. Rev. Neurol. 10, 74-81 (2014).

3. Krumm, N., O'Roak, B. J., Shendure, J. \& Eichler, E. E. A de novo convergence of autism genetics and molecular neuroscience. Trends Neurosci. 37, 95-105 (2014).
4. Sudhof, T. C. Neuroligins and neurexins link synaptic function to cognitive disease. Nature 455, 903-911 (2008).

5. Ting, J. T., Peca, J. \& Feng, G. Functional consequences of mutations in postsynaptic scaffolding proteins and relevance to psychiatric disorders. Annu. Rev. Neurosci. 35, 49-71 (2012).

6. Jiang, Y. H. \& Ehlers, M. D. Modeling autism by SHANK gene mutations in mice. Neuron 78, 8-27 (2013).

7. Silverman, J. L., Yang, M., Lord, C. \& Crawley, J. N. Behavioural phenotyping assays for mouse models of autism. Nat. Rev. Neurosci. 11, 490-502 (2010).

8. Bagni, C. \& Oostra, B. A. Fragile X syndrome: from protein function to therapy. Am. J. Med. Genet. A 161A, 2809-2821 (2013).

9. Kleijer, K. T. et al. Neurobiology of autism gene products: towards pathogenesis and drug targets. Psychopharmacology (Berl) 231, 1037-1062 (2014).

10. Darnell, J. C. \& Klann, E. The translation of translational control by FMRP: therapeutic targets for FXS. Nat. Neurosci. 16, 1530-1536 (2013).

11. Ebert, D. H. \& Greenberg, M. E. Activity-dependent neuronal signalling and autism spectrum disorder. Nature 493, 327-337 (2013).

12. Zoghbi, H. Y. \& Bear, M. F. Synaptic dysfunction in neurodevelopmental disorders associated with autism and intellectual disabilities. Cold Spring Harb. Perspect. Biol. 4 (2012).

13. Ehninger, D. \& Silva, A. J. Rapamycin for treating tuberous sclerosis and autism spectrum disorders. Trends Mol. Med. 17, 78-87 (2011).

14. Ghosh, A., Michalon, A., Lindemann, L., Fontoura, P. \& Santarelli, L. Drug discovery for autism spectrum disorder: challenges and opportunities. Nat. Rev. Drug Disc. 12, 777-790 (2013).

15. Persico, A. M. \& Bourgeron, T. Searching for ways out of the autism maze: genetic, epigenetic and environmental clues. Trends Neurosci. 29, 349-358 (2006).

16. Grabrucker, A. M. Environmental factors in autism. Front. Psychiatry 3, 118 (2012).

17. Grabrucker, A. M., Rowan, M. \& Garner, C. C. Brain-delivery of zinc-ions as potential treatment for neurological diseases: mini review. Drug Deliv. Lett. 1, 13-23 (2011)

18. Yasuda, H., Yoshida, K., Yasuda, Y. \& Tsutsui, T. Infantile zinc deficiency: association with autism spectrum disorders. Sci. Rep. 1, 129 (2011).

19. Grabrucker, S. et al. Zinc deficiency dysregulates the synaptic ProSAP/Shank scaffold and might contribute to autism spectrum disorders. Brain 137, 137-152 (2014).

20. Russo, A. J. \& Devito, R. Analysis of copper and zinc plasma concentration and the efficacy of zinc therapy in individuals with Asperger's syndrome, pervasive developmental disorder not otherwise specified (pdd-nos) and autism. Biomark Insights 6, 127-133 (2011).

21. Sensi, S. L., Paoletti, P., Bush, A. I. \& Sekler, I. Zinc in the physiology and pathology of the CNS. Nat. Rev. Neurosci. 10, 780-791 (2009).

22. Gundelfinger, E. D., Boeckers, T. M., Baron, M. K. \& Bowie, J. U. A role for zinc in postsynaptic density asSAMbly and plasticity? Trends Biochem. Sci. 31, 366-373 (2006).

23. Sensi, S. L. et al. The neurophysiology and pathology of brain zinc. J. Neurosci. 31, 16076-16085 (2011).

24. Vergnano, A. M. et al. Zinc dynamics and action at excitatory synapses. Neuron 82, 1101-1114 (2014).

25. Kim, T. Y., Hwang, J. J., Yun, S. H., Jung, M. W. \& Koh, J. Y. Augmentation by zinc of NMDA receptor-mediated synaptic responses in CA1 of rat hippocampal slices: mediation by Src family tyrosine kinases. Synapse 46, 49-56 (2002).

26. Huang, Y. Z., Pan, E., Xiong, Z. Q. \& McNamara, J. O. Zinc-mediated transactivation of TrkB potentiates the hippocampal mossy fiber-CA3 pyramid synapse. Neuron 57, 546-558 (2008).

27. Manzerra, P. et al. Zinc induces a Src family kinase-mediated up-regulation of NMDA receptor activity and excitotoxicity. Proc. Natl Acad. Sci. USA 98, 11055-11061 (2001).

28. Sheng, M. \& Kim, E. The shank family of scaffold proteins. J. Cell Sci. 113, 1851-1856 (2000).

29. Boeckers, T. M., Bockmann, J., Kreutz, M. R. \& Gundelfinger, E. D. ProSAP/ Shank proteins-a family of higher order organizing molecules of the postsynaptic density with an emerging role in human neurological disease. J. Neurochem. 81, 903-910 (2002).

30. Grabrucker, A. M. et al. Concerted action of zinc and ProSAP/Shank in synaptogenesis and synapse maturation. EMBO J. 30, 569-581 (2011).

31. Leblond, C. S. et al. Genetic and functional analyses of SHANK2 mutations suggest a multiple hit model of autism spectrum disorders. PLoS Genet. 8, e1002521 (2012).

32. Berkel, S. et al. Mutations in the SHANK2 synaptic scaffolding gene in autism spectrum disorder and mental retardation. Nat. Genet. 42, 489-491 (2010).

33. Durand, C. M. et al. Mutations in the gene encoding the synaptic scaffolding protein SHANK3 are associated with autism spectrum disorders. Nat. Genet. 39, 25-27 (2007). 
34. Pinto, D. et al. Functional impact of global rare copy number variation in autism spectrum disorders. Nature 466, 368-372 (2010).

35. Leblond, C. S. et al. Meta-analysis of SHANK mutations in autism spectrum disorders: a gradient of severity in cognitive impairments. PLoS Genet. 10, e1004580 (2014).

36. Moessner, R. et al. Contribution of SHANK3 mutations to autism spectrum disorder. Am. J. Hum. Genet. 81, 1289-1297 (2007).

37. Verpelli, C. et al. Importance of Shank3 protein in regulating metabotropic glutamate receptor 5 (mGluR5) expression and signaling at synapses. J. Biol. Chem. 286, 34839-34850 (2011).

38. Berkel, S. et al. Inherited and de novo SHANK2 variants associated with autism spectrum disorder impair neuronal morphogenesis and physiology. Hum. Mol. Genet. 21, 344-357 (2011)

39. Arons, M. H. et al. Autism-associated mutations in ProSAP2/Shank3 impair synaptic transmission and neurexin-neuroligin-mediated transsynaptic signaling. J. Neurosci. 32, 14966-14978 (2012).

40. Bozdagi, O. et al. Haploinsufficiency of the autism-associated Shank3 gene leads to deficits in synaptic function, social interaction, and social communication. Mol. Autism 1, 15 (2010).

41. Kouser, M. et al. Loss of predominant Shank3 isoforms results in hippocampusdependent impairments in behavior and synaptic transmission. J. Neurosci. 33, 18448-18468 (2013)

42. Peca, J. et al. Shank3 mutant mice display autistic-like behaviours and striatal dysfunction. Nature 472, 437-442 (2011).

43. Schmeisser, M. J. et al. Autistic-like behaviours and hyperactivity in mice lacking ProSAP1/Shank2. Nature 486, 256-260 (2012).

44. Wang, X. et al. Synaptic dysfunction and abnormal behaviors in mice lacking major isoforms of Shank3. Hum. Mol. Genet. 20, 3093-3108 (2011)

45. Won, H. et al. Autistic-like social behaviour in Shank2-mutant mice improved by restoring NMDA receptor function. Nature 486, 261-265 (2012)

46. Yang, M. et al. Reduced excitatory neurotransmission and mild autism-relevant phenotypes in adolescent Shank3 null mutant mice. J. Neurosci. 32, 6525-6541 (2012).

47. Wang, X., Xu, Q., Bey, A. L., Lee, Y. \& Jiang, Y. H. Transcriptional and functional complexity of Shank3 provides a molecular framework to understand the phenotypic heterogeneity of SHANK3 causing autism and Shank3 mutant mice. Mol. Autism 5, 30 (2014)

48. Zhu, L. et al. Epigenetic dysregulation of SHANK3 in brain tissues from individuals with autism spectrum disorders. Hum. Mol. Genet. 23, 1563-1578 (2014).

49. Han, K. et al. SHANK3 overexpression causes manic-like behaviour with unique pharmacogenetic properties. Nature 503, 72-77 (2013).

50. Huang, T. N. et al. Tbr1 haploinsufficiency impairs amygdalar axonal projections and results in cognitive abnormality. Nat. Neurosci. 17, 240-247 (2014).

51. Chuang, H. C., Huang, T. N. \& Hsueh, Y. P. Neuronal excitation upregulates Tbr1, a high-confidence risk gene of autism, mediating Grin2b expression in the adult brain. Front. Cell. Neurosci. 8, 280 (2014).

52. Bareggi, S. R. \& Cornelli, U. Clioquinol: review of its mechanisms of action and clinical uses in neurodegenerative disorders. CNS Neurosci. Ther. 18, 41-46 (2012).

53. Grabrucker, A. M. A role for synaptic zinc in ProSAP/Shank PSD scaffold malformation in autism spectrum disorders. Dev. Neurobiol. 74, 136-146 (2014).

54. Cole, T. B., Wenzel, H. J., Kafer, K. E., Schwartzkroin, P. A. \& Palmiter, R. D. Elimination of zinc from synaptic vesicles in the intact mouse brain by disruption of the ZnT3 gene. Proc. Natl Acad. Sci. USA 96, 1716-1721 (1999).

55. Izumi, Y., Auberson, Y. P. \& Zorumski, C. F. Zinc modulates bidirectional hippocampal plasticity by effects on NMDA receptors. J. Neurosci. 26, 7181-7188 (2006).

56. Frederickson, C. J. et al. Concentrations of extracellular free zinc (pZn)e in the central nervous system during simple anesthetization, ischemia and reperfusion. Exp. Neurol. 198, 285-293 (2006).

57. Takeda, A. Zinc signaling in the hippocampus and its relation to pathogenesis of depression. J. Trace Elem. Med. Biol. 26, 80-84 (2012).

58. Takeda, A. et al. Transient increase in $\mathrm{Zn} 2+$ in hippocampal CA1 pyramidal neurons causes reversible memory deficit. PloS ONE 6, e28615 (2011).
59. Salter, M. W. \& Kalia, L. V. Src kinases: a hub for NMDA receptor regulation. Nat. Rev. Neurosci. 5, 317-328 (2004).

60. Yu, X. M., Askalan, R., Keil, 2nd G. J. \& Salter, M. W. NMDA channel regulation by channel-associated protein tyrosine kinase Src. Science 275, 674-678 (1997).

61. Lu, Y. M., Roder, J. C., Davidow, J. \& Salter, M. W. Src activation in the induction of long-term potentiation in CA1 hippocampal neurons. Science 279, 1363-1367 (1998).

62. Frederickson, C. J., Koh, J. Y. \& Bush, A. I. The neurobiology of zinc in health and disease. Nat. Rev. Neurosci. 6, 449-462 (2005).

63. Adlard, P. A. et al. Rapid restoration of cognition in Alzheimer's transgenic mice with 8-hydroxy quinoline analogs is associated with decreased interstitial Abeta. Neuron 59, 43-55 (2008).

64. Lee, E. J., Choi, S. Y. \& Kim, E. NMDA receptor dysfunction in autism spectrum disorders. Curr. Opin. Pharmacol. 20C, 8-13 (2015).

65. Paoletti, P., Bellone, C. \& Zhou, Q. NMDA receptor subunit diversity: impact on receptor properties, synaptic plasticity and disease. Nat. Rev. Neurosci. 14, 383-400 (2013).

66. LeBlanc, J. J. \& Fagiolini, M. Autism: a 'critical period' disorder? Neural. Plast. 2011, 921680 (2011).

67. Bush, A. I. The metal theory of Alzheimer's disease. J. Alzheimers Dis. 33(Suppl 1): S277-S281 (2013).

68. Lei, P. et al. Tau deficiency induces parkinsonism with dementia by impairing APP-mediated iron export. Nat. Med. 18, 291-295 (2012).

69. Nguyen, T., Hamby, A. \& Massa, S. M. Clioquinol down-regulates mutant huntingtin expression in vitro and mitigates pathology in a Huntington's disease mouse model. Proc. Natl Acad. Sci. USA 102, 11840-11845 (2005).

70. Coyle, J. T., Basu, A., Benneyworth, M., Balu, D. \& Konopaske, G. Glutamatergic synaptic dysregulation in schizophrenia: therapeutic implications. Handb. Exp. Pharmacol. 267-295 (2012).

\section{Acknowledgements}

The work was supported by Academia Sinica and Ministry of Science and Technology, Taiwan (MOST 103-2321-B-001-002 to Y.-P.H. and 102-2811-B-001-060 to T.-N.E.H.) the NRF (National Research Foundation of Korea) grant funded by the Korean Government (NRF-2013-Fostering Core Leaders of the Future Basic Science Program to C.C.) and the Institute for Basic Science (IBS-R002-D1 to E.K.).

\section{Author contributions}

H.L. performed immunoblot experiments and imaging analyses; C.C. performed NMDAfEPSP experiments; W.S. performed behavioural and $\mathrm{Zn}$ staining experiments; K.K. performed behavioural experiments; T.-N.E.H. performed behavioural experiments for Tbr1 mice. E.-J.L. performed the majority of electrophysiological analyses and all the other experiments; and J.-Y.K., Y.-P.H. and E.K. supervised the project and wrote the manuscript.

\section{Additional information}

Supplementary Information accompanies this paper at http://www.nature.com/ naturecommunications

Competing financial interests: The authors declare no competing financial interests

Reprints and permission information is available online at http://npg.nature.com/ reprintsandpermissions/

How to cite this article: Lee, E. -J. et al. Trans-synaptic zinc mobilization improves social interaction in two mouse models of autism through NMDAR activation. Nat. Commun 6:7168 doi: 10.1038/ncomms8168 (2015).

This work is licensed under a Creative Commons Attribution 4.0 International License. The images or other third party material in this article are included in the article's Creative Commons license, unless indicated otherwise in the credit line; if the material is not included under the Creative Commons license, users will need to obtain permission from the license holder to reproduce the material To view a copy of this license, visit http://creativecommons.org/licenses/by/4.0/ 